\title{
Sustainable Investing and the Cross-Section of Maximum Drawdown*
}

\author{
Lisa R. Goldberg and Saad Mouti \\ Consortium for Data Analytics in Risk, University of California, Berkeley
}

May 15, 2019

\begin{abstract}
We use supervised learning to identify factors that predict the cross-section of maximum drawdown for stocks in the US equity market. Our data run from January 1980 to June 2018 and our analysis includes ordinary least squares, penalized linear regressions, tree-based models, and neural networks. We find that the most important predictors tended to be consistent across models, and that non-linear models had better predictive power than linear models. Predictive power was higher in calm periods than stressed periods, and environmental, social, and governance indicators augmented predictive power for non-linear models.
\end{abstract}

\section{Introduction}

We apply a variety supervised learning models to forecast maximum drawdown - the largest decline in cumulative return over a fixed period. As predictors, we use standard accounting ratios and factors, sector information, and environmental, social and governance (ESG) indicators. We look specifically at whether or not ESG indicators augment the predictive power of our methods.

\subsection{Motivation}

The search for factors that drive return and risk of stocks has been the focus of the asset pricing theory for decades. While the capital asset pricing model (CAPM), see [Sharpe, 1964] and [Lintner, 1965], is probably the first model to address this question and states that a stocks' returns are explained by their market risk through the betas, empirical findings suggested that this model is incomplete. Despite decades of research and hundreds of papers, a thorough understanding of the drivers of cross-sectional variation of expected returns eludes us. The Fama and French three-factor model, see [Fama and French, 1992], and the Carhart four-factor model, see [Carhart, 1997], are well accepted and go a long way in capturing this variation. They demonstrate that size, market-tobook ratio, and momentum are significant drivers of asset returns, and they complement the CAPM. Nevertheless, new empirical studies continue to emerge, and they document a growing number of factors, see [Fama and French, 2008] and [Harvey et al., 2015] for an overview, or [Green et al., 2013] and [Feng et al., 2017] for an extensive factors mining. In a recent paper, [Gu et al., 2018] investigate the use of supervised learning models to explore further the predictive power of firms' characteristics, and find an advantage in using non-linear models.

${ }^{*}$ We thank Robert Anderson, Wachi Bandara, Jeff Bohn, Claudia Bolli, Dangxin Chen, Peter Clark, Jeremy Evnine, Tingyue Gan, Nicholas L. Gunther, Jim Hawkey, Kathleen Houssels, Paul Jung, Ran Leshem, Raymond Leung, Shailee Pradhan, and different participants of the risk seminar at UC Berkeley for their comments and suggestions. We are grateful to the Swiss Re Institute for funding through the Consortium for Data Analytics in Risk (CDAR), and to OWL Analytics for data. 
The use of firm characteristics to forecast attributes of individual stocks is not restricted to return. For example, Barra models, which are standard in industry, use firm characteristics to estimate stock covariance matrices, see [Bayraktar et al., 2014]. They decompose the covariance matrix of security returns using the estimation of sensitivities of the securities to the common factors, the covariance matrix of factors, and the variances of security specific returns. [Vozlyublennaia, 2013] investigate the effect of firm characteristics in the dynamics of idiosyncratic risk. They find that firm characteristics can be used in the analysis of the differences in risk across securities. [Herskovic et al., 2016] also show that a firm's idiosyncratic volatility obeys a strong factor structure. In our paper, we are also interested in firm's risk, although we choose a different measure, maximum drawdown.

Maximum drawdown has received extensive attention in the literature on financial markets. However, most of the papers are theoretical. [Taylor, 1975] elucidates properties of maximum drawdown under the assumption of a Brownian diffusion for the underlying process, Magdon-Ismail et al., 2004] on the other hand gives a series representation of the maximum drawdown distribution and estimate its expected value. A second body of literature addresses portfolio construction and formalizes some risk measures based on maximum drawdown, see [Chekhlov et al., 2004], [Heidorn et al., 2009], or [Goldberg and Mahmoud, 2016]. Our paper tackles different questions than the papers cited in that our analysis is empirical and aims to understand what factors drive maximum drawdowns of individual stocks. Closer to this line of inquiry is [Daniel and Moskowitz, 2016], who look at how momentum strategies perform following drawdowns, and [Plastira, 2014] who studies performance rankings of popular size, value, reversal and momentum portfolios using drawdown-based portfolio performance measures.

This project began with the question of whether sustainability metrics predict stock risk. Sustainability metrics include a set of quality factors that specialized agencies assign to companies based on their environmental, social, and governance (ESG) strengths and weaknesses. Although sustainable investing was a niche topic a few years ago, it has become mainstream for many asset managers and mutual funds.According to a late 2018 Forbes article, sustainable responsible investing (SRI) attained $\$ 12$ trillion in assets under management in the US alone. It is no surprise that it has also become a key topic in financial research. SRI can be roughly defined as "an investment process that integrates social, environmental, and ethical considerations into investment decision making", see [Renneboog et al., 2008]. This involves screening companies with high corporate social responsibility by evaluating on the basis of ESG criteria. While the question of non-financial utility is undisputed, whether these ESG issues have a material economic impact is still an ongoing debate.

The main question that animates the dialog around ethical investing is whether companies that "do good" also "do well." This statement suggests that companies with superior ESG performance generate higher financial performance. In that sense, a wide range of literature explores the link between sustainability metrics and several dimensions of performance and risk. [Preston and O'Bannon, 1997] explore the possible empirical association between social and financial performance (through return on assets and other measures) in longitudinal data. Their sustainability data are based on a Fortune magazine reputation ratings for individual companies. They find that positive synergies explain social-financial performance correlations. [Khan et al., 2016] using KLD ${ }^{1}$ data and explore the question of materiality, i.e., a classification that maps different sustainable indicators as material for different industries. Using both portfolio and firm-level regressions, they find that firms with good ratings significantly outperform firms with poor ratings. Immaterial sustainability issues, on the other hand, do not improve financial performance.

An opposing point of view is "doing good but not well". This view is linked to the "managerial

\footnotetext{
${ }^{1}$ Founded in 1988, KLD Research \& Analytics, Inc. provides performance benchmarks, corporate accountability research, and consulting services. The company offers environmental, social, and governance research for institutional investors.
} 
opportunism hypothesis," which suggests that managers tend to maximize their gains and that socially responsible activity may cost resources to the firm, putting in relative disadvantage to firms that invest less in sustainability issues, see [Aupperle et al., 1985]. In that sense, [Brammer et al., 2006] and [Hong and Kacperczyk, 2009] show a higher performance for portfolios with low sustainability performance compared to their peers with high sustainability performance.

Our paper, on the other hand, explores the question of how sustainability issues impact financial risk through maximum drawdown. We ask whether ESG data enhance our ability to predict future maximum drawdown. Previous literature explores similar question by testing for a relationship between sustainability measures and firm systematic risk. In this line of inquiry, [Jo and $\mathrm{Na}, 2012$ ] and [Benlemlih et al., 2018] use the CAPM beta as the measure of a firm's systematic risk. While the first of the two papers focuses on 'controversial industries', both studies find a strong negative link between corporate social performance and systematic risk. [Cholleta and Sandwidi, 2018] consider, in addition to systematic risk, specific and total risk, translated respectively by the standard deviation of residuals from the CAPM model and the volatility of stocks. They find that a firm's good social and governance performance reduces its financial risk.

\subsection{Contributions}

While this research began with the assessment of ESG impact on stock performance, the contributions of this paper go beyond that. We identify determinants of the cross-section drawdown using firm's quantitative and qualitative characteristics. We perform a pooled regression analysis using firm-level characteristics and nine regression algorithms in supervised statistical learning and compare the performance of these methods based on their out-of-sample performance.

If stock returns are known to be difficult to predict in general and incorporate a low signal to noise ratio, maximum drawdown, which measures a firm's risk of successive negative returns over a period of time, has higher predictability across firms. The first analysis seeks to find if stock level characteristics explain maximum drawdown over a large data set that includes 2008 financial without ESG variables. We then split our data to account for ESG variables and repeat the analysis. We find that maximum drawdown gives high predictability results in both cases. We are able to obtain both high predictability and economically meaningful results.

Our empirical analysis sheds light to what features are most valuable to forecasting its future performance and drawdown. By applying nine regression methods, both linear models and non-linear ones on the field of machine learning, and analyzing what features these models give higher forecasting weight, we are able to classify the most dominant variables and those with the lowest impact. We take advantage of this framework to find if there is a relationship between environmental, social, and governance level of stocks and their future performance.

\subsection{Empirical findings}

We rely on monthly stock data from January 1980 until June 2017 with a total of 450 months, and an average of 3,700 individual stocks per month. The predictive signals are lagged firm characteristics with a total of 96 signals, 16 of which are ESG related. We conduct one analysis using all the variables at once, and we then reduce the number of predictors to a few ones that are used mainly in the industry. Due to the co-linearity between ESG scores, we group them into three sets; one where we include the "raw" ESG scores, one where we include the three aggregated ones (E, S, and G), and one with the single average ESG score. Finally, we compare the forecast of a set of linear and nonlinear supervised learning algorithms. The empirical findings of our analysis are summarized in the following. 
in asset pricing theory. Supervised learning approaches usually aim to predict an output from a number of input variables. Linear regressions, either ordinary or penalized, and dimension reduction methods are limited to linear relationships. The advantage of more complex models used in machine learning like tree-based models and neural networks, is that they can overcome this limitation and account for non-linear relationships without increasing the dimension of the problem (by introducing new variables). Our results show that non-linear models improve the performance compared.

Maximum drawdown was predictable. Empirical results suggest that firm characteristics can predict the cross-sectional variation of maximum drawdown. We report two performance metrics; the mean absolute error (MAE), and Lin's concordance correlation coefficient CCC. The first measures accuracy and the second measures agreement between the realized and predicted $M D D$. We find that MAE averaged approximately 14.5\% between March 2007 and June 2017, and it reached a high of $30 \%$ during the 2008 crisis. CCC, on the other hand, averaged $45 \%$ and reached $15 \%$ during the 2008 crisis. However, the positive sign of CCC suggests that even during low-performance periods, there was a positive relationship between the realized and predicted values of $M D D$.

The set of dominant features was largely consistent across models. Based on a sensitivity analysis that ranks variables by their predictive power, the top five variables in most models were bid-ask spread, volatility, beta, size, and momentum when ESG indicators were excluded.

Environmental, social, and governance scores improved the predictability of maximum drawdown. ESG scores improved the predictability of $M D D$ over a limited test period, June 2014 to June 2017, and relying only on the non-linear models. The limitations were imposed by the relatively short data history of ESG indicators. We find that non-linear models placed ESG indicators among the top 20 predictors. Moreover, we find that companies with a higher ESG score are more likely to have lower drawdowns.

\section{Methodology}

One of the goals of this paper is to compare the ability of different supervised learning methods to predict next period maximum drawdown from an observed set of quantitative and qualitative firm attributes. For completeness, we summarize the (well-known) methods used in the analysis here.

All the methods we consider follow the general equations:

$$
y_{i, t+1}=\mathbb{E}_{t}\left[y_{i, t+1}\right]+\varepsilon_{i, t+1},
$$

and

$$
\mathbb{E}_{t}\left[y_{i, t+1}\right]=f^{*}\left(x_{i, t}\right),
$$

where $f^{*}$ defines a general mapping function, $y_{i t+1}$ denotes drawdown of firm $i$ over the period from time $t$ to time $t+1, x_{i, t}=\left[x_{i, t}^{1}, \ldots, x_{i, t}^{J}\right]$ is a vector of realizations of firm characteristics for a stock $i$ at time $t$, and $\varepsilon_{i, t+1}$ an error term.

The relationship expressed in formulas (1) and (2) mirrors the asset pricing relationships specified in countless papers in the finance literature. In those papers, the dependent variable is usually excess return over the risk-free rate or another benchmark. 


\subsection{The Models}

\section{Linear models}

The first set of methods we use are linear regression models, in which the mapping function $f$ is a linear combination of the input vector $x_{i, t}=\left[x_{i, t}^{1}, x_{i, t}^{2}, \ldots, x_{i, t}^{J}\right]$ :

$$
\begin{aligned}
f\left(x_{i, t} ; \beta\right) & =x_{i, t}^{\prime} \beta \\
& =\beta_{0}+\sum_{j=1}^{J} \beta_{j} x_{i, t}^{j}
\end{aligned}
$$

where the $\beta$ is the parameter vector and $x_{i, t}^{\prime}$ is the transpose of $x_{i, t}$ the vector of the firm characteristics for stock $i$ at time $t$.

\section{Ordinary Least Square Regression}

To find the regression parameters $\beta_{0}, \beta_{1}, \ldots, \beta_{J}$, linear regression minimizes the square of error between the observation $y_{i, t+1}$, and the prediction $f\left(x_{i, t}\right)$ :

$$
\beta=\underset{\beta}{\arg \min } \frac{1}{N T} \sum_{t=1}^{T} \sum_{i=1}^{N_{t}}\left(y_{i, t+1}-f\left(x_{i, t+1}\right)\right)^{2}
$$

When the number of input features is large or when the regressors are correlated, linear regression can lead to overfitting and leads to spurious coefficients.

\section{Penalized regression techniques: Lasso, Ridge, and Elastic Net}

Penalized regression models aim to create a more robust output model in the presence of a large number of potentially correlated variables. They are a simple modification of the ordinary linear regression that introduces a regularization term in the optimization problem - a technique in Machine Learning that aims at reducing the out-of-sample forecasting error by penalizing the coefficients. In this paper, we explore the three main ones: Lasso, Ridge and Elastic Net.

- The first penalized regression technique called Lasso, for "least absolute shrinkage and selection operator", see [Tibshirani, 1996], is based on a penalty term equal to the absolute value of the beta coefficients:

$$
\beta=\underset{\beta}{\arg \min } \frac{1}{N T} \sum_{t=1}^{T} \sum_{i=1}^{N_{t}}\left(y_{i, t+1}-f\left(\boldsymbol{x}_{i, t} ; \beta\right)\right)^{2}+\lambda \sum_{j=1}^{J}\left|\beta_{j}\right|
$$

where $\lambda$ is a non-negative hyperparameter.

- Ridge regression, see [Hoerl, 1962], adds a penalty related to the square of the magnitude of the coefficients called $\ell_{2}$ regularization and solves the following objective function:

$$
\beta=\underset{\beta}{\arg \min } \frac{1}{N T} \sum_{t=1}^{T} \sum_{i=1}^{N_{t}}\left(y_{i, t+1}-f\left(\boldsymbol{x}_{\boldsymbol{i}, t} ; \beta\right)\right)^{2}+\lambda \sum_{j=1}^{J} \beta_{j}^{2}
$$

where $\lambda$ is a non-negative hyperparameter. 
- Elastic Net, see [Zou and Hastie, 2005], uses an intermediate objective between the Lasso and Ridge:

$$
\beta=\underset{\beta}{\arg \min } \frac{1}{N T} \sum_{t=1}^{T} \sum_{i=1}^{N_{t}}\left(y_{i, t+1}-f\left(\boldsymbol{x}_{\boldsymbol{i}, t} ; \beta\right)\right)^{2}+\lambda_{1} \sum_{j=1}^{J}\left|\beta_{j}\right|+\lambda_{1} \sum_{j=1}^{J} \beta_{j}^{2}
$$

where $\lambda_{1}, \lambda_{2}$ are two non-negative hyperparameters.

By setting the parameter $\lambda=0$ (resp. $\lambda_{1}=\lambda_{2}=0$ ), we recover the ordinary linear regression. Moreover, as $\lambda$ (resp. $\lambda_{1}$ and $\lambda_{2}$ ) increases, we choose a smaller set of predictors by decreasing the value of the coefficients and shrinking the least relevant ones.

\section{Dimension Reduction}

Dimension reduction techniques decrease the number of features in a data set without discarding salient information. In contrast to penalized regression methods, which discard weak regressors by setting their loadings to zero, dimension reduction techniques form uncorrelated linear combination of the predictors for the purpose reducing noise and concentrating signal. In our analysis, we rely on two widely used methods, principal component regression, and partial least squares.

Principal Component Regression (PCR) is a two-step procedure. The first step is a principal component analysis (PCA) that combines the independent variables into a set of leading components ranked by their explained variance. The predicted variable plays no role in this step. The second step is a simple linear regression on the leading components. PCA is one of the most widely used dimensionality reduction techniques, and it dates back to [Pearson, 1901]. The key idea is to find a new coordinate system in which the input data can be expressed with fewer variables without a significant error.

Unlike PCR, in which the two steps are performed separately, a partial least squares (PLS) regression combines dimension reduction and regression by directly taking account of the covariance of the predictors with the target prediction. This is carried out by estimating, for each predictor $p$, a univariate return prediction coefficient via OLS. This coefficient $\varphi_{p}$ represents the partial sensitivity of returns to a given predictor $p$. Predictors are then averaged into a single aggregate component with weights proportional to $\varphi_{p}$, which would give the strongest univariate predictors the highest weights. Then the target and all predictors are orthogonalized with respect to previously constructed components, and the procedure is repeated on the orthogonalized set. The procedure stops when the desired number of components is obtained.

More formally, we write the linear model in its vectorized version,

$$
R=Z \theta+E
$$

where $R$ is the $N T \times 1$ vector of $r_{i, t+1}, Z$ is the $N T \times P$ matrix of stacked predictors $z_{i, t}$, and $E$ is a $N T \times 1$ vector of residuals $\varepsilon_{i, t+1}$.

The linear model given above is re-written for the set of reduced predictors:

$$
R=\left(Z \Omega_{K}\right) \theta_{K}+E,
$$

where $K$ is the number of reduced predictors corresponding to a linear combination of the initial ones, $\Omega_{K}$ is the $P \times K$ matrix with columns $w_{1}, w_{2}, \ldots, w_{K}$, where $w_{j}$ for $j \in 1, \ldots, K$ is the set of linear combination weights used to create the $j^{\text {th }}$ predictive components, and $Z \Omega_{K}$ is the reduced version of the original predictor set. 


\section{Regression Trees and Random Forests}

Decision trees are among the simplest non-linear models that rely on a tree structure to approach the outcome.

We can express the prediction of a tree with $M$ terminal nodes and depth $L$ as:

$$
f\left(\boldsymbol{x}_{\boldsymbol{i}, t} ; \theta, M, L\right)=\sum_{m=1}^{M} \theta_{m} \mathbb{1}_{\boldsymbol{x}_{i, t \in} \in C_{m}(L)},
$$

where each $C_{m}(L)$ is one of the $M$ partitions of the data.

The algorithm that fits the decision sequentially splits subsets of the data in two on the basis of one of the predictors. The split at each step is chosen to optimize a loss function defined in terms of impurities in the child nodes. Impurity is typically measured in terms of the Gini index or entropy. To prevent overfitting and to ensure the tree is interpretable, different criteria can be used like the maximum depth of the tree or node size.

A random forests averages the output of many decision trees. Each decision tree is fit on a small subset of training examples or constrained to use a subset of input features. Doing so increases the bias relative to a simple decision tree, but decreases the variance, see [Breiman, 2001] for more details.

Formally, if a regression tree has $L$ leaves and accepts a vector of size $m$ as input, then we can define a function $q: \mathbb{R}^{m} \rightarrow\{1, \ldots, T\}$ that maps an input to a leaf index. If we denote the score at a leaf by the function $w$, then we can define the $k$-th tree (within the ensemble of trees considered) as a function $f_{k}(x)=w_{q(x)}$, where $w \in \mathbb{R}^{T}$. For a training set of size $n$ with samples given by $\left(x_{i}, y_{i}\right), x_{i} \in \mathbb{R}^{m}, y_{i} \in \mathbb{R}$, a tree ensemble model uses $K$ additive functions to predict the output as follows:

$$
\hat{y}_{i, t+1}=f\left(\boldsymbol{x}_{\boldsymbol{i}, \boldsymbol{t}}\right)=\sum_{k=1}^{K} f_{k}\left(\boldsymbol{x}_{\boldsymbol{i}, \boldsymbol{t}}\right) .
$$

\section{Extreme Gradient Boosting}

The term 'boosting' refers to the technique of iteratively combining weak learners (i.e., algorithms with weak predictive power) to form an algorithm with strong predictive power. Boosting starts with a weak learner like a regression tree algorithm, and records the error between the learner's predictions and the actual output. At each stage of the iteration, it uses the error to improve the weak learner from the previous iteration step. If the error term is calculated as a negative gradient of a loss function, the method is called 'gradient boosting.' Extreme gradient boosting (or XGBoost) refers to the optimized implementation in [Chen and Guestrin, 2016].

Formally, the model uses $K$ additive functions to predict the output as follows:

$$
\hat{y}_{i, t+1}=f\left(\boldsymbol{x}_{\boldsymbol{i}, \boldsymbol{t}}\right)=\sum_{k=1}^{K} f_{k}\left(\boldsymbol{x}_{\boldsymbol{i}, \boldsymbol{t}}\right),
$$

where we take $f_{k}(x)=w_{q(x)}\left(q: \mathbb{R}^{m} \rightarrow T, w \in \mathbb{R}\right)$ from the space of regression tree. $q$ represents the structure of each tree that maps an example of the data set to the corresponding leaf index, $T$ is the number of leaves in the tree, and each $f_{k}$ corresponds to an independent tree structure $q$ and leaf weight $w$. 
To learn the set of functions in the model, the regularized objective is defined as:

$$
\mathcal{L}(f)=\sum_{t} \sum_{i} l\left(\hat{y}_{i, t+1}, y_{i, t+1}\right)+\sum_{k} \Omega\left(f_{k}\right)
$$

where $\Omega(f)=\gamma T+\frac{1}{2} \lambda\|w\|^{2}$.

The model is then optimized in an additive manner. If $\hat{y}_{i}^{(t)}$ is the prediction for the $i$-th training example at the $t$-th stage of boosting iteration, then we seek to augment our ensemble collection of trees by a function $f_{t}$ that minimizes the following objective:

$$
\mathcal{L}^{(t)}=\sum_{i=1}^{n} l\left(y_{i}, \hat{y}_{i}^{(t-1)}+f_{t}\left(x_{i}\right)\right)+\Omega\left(f_{t}\right) .
$$

The objective function is approximated by a second-order Taylor expansion and then optimized (see [Chen and Guestrin, 2016] for details and calculation steps). To prevent overfitting, XGBoost uses shrinkage and feature sub-sampling.

\section{Artificial Neural Networks: The Multi-Layer Perceptron}

Artificial neural networks (ANN) are a set of algorithms inspired by the human brain and designed to recognize patterns. The idea behind such a framework is to represent complex non-linear functions by connecting simple processing units into a neural network, each of which computes a linear function, possibly followed by a non-linearity.

A neuron-like processing unit is given by:

$$
a=\phi\left(\sum_{j} w_{j} x_{j}+b\right)
$$

where the $x_{j}$ 's are the inputs to the unit, the $w_{j}$ 's are the weights, $b$ is the bias, $\phi$ is a non-linear activation function, and $a$ is the unit's activation. An activation function is a function used to transform a trigger level of a unit (neuron) into an output signal. Examples of such activation functions are:

- The identity activation function: $\phi(x)=x$.

- The logistic activation function: $\phi(x)=\frac{1}{\left(1+e^{-x}\right)}$.

- The hyperbolic tan function 'Tanh': $f(x)=\tanh (x)$.

- The rectifier linear unit function 'ReLu': $f(x)=\max (0, x)$.

The combination of a set of these units is what forms the neural network. Each unit performs a simple function, but in aggregate, the units can do more complex computations. In our analysis we apply a variant of the simple feed-forward neural network, that is, the multi-layer perceptron. In such a model, the units are arranged in an acyclic graph and calculations are done sequentially (in contrast to a recurrent neural network, where the graph can have cycles).

The multi-layer perceptron (MLP), as shown in Figure 1, has units that are arranged in a set of layers that contain identical units. In MLP, the network is fully connected, i.e., every unit in one layer is connected to every unit in the next layer. The first input takes the values of the input features. The last output layer has a single unit in the case of regression. The intervening hidden layers are mysterious because we do not know ahead of time what their units should compute. The number of layers is known as the depth, and the number of units in a layer is known as the width. "Deep learning" refers to training neural networks with many layers. 


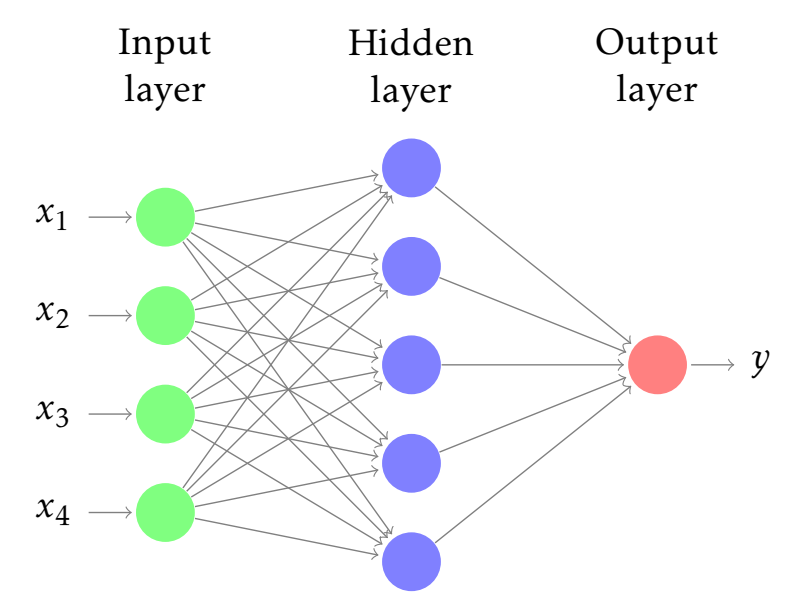

Figure 1: An Artificial Neural Network with one hidden layer

We denote the input units by $x_{j}$, the output unit by $y$, and the $\ell$-th hidden layer by $h_{i}^{(\ell)}$. MLP is fully connected so each unit receives input from all the units in the previous layer. This implies that each unit has its own bias and a weight is associated to every pair of units in consecutive layers:

$$
\begin{aligned}
h_{i}^{(1)} & =\phi^{(1)}\left(\sum_{j} w_{i j}^{(1)} x_{j}+b_{i}^{(1)}\right) \\
h_{i}^{(1)} & =\phi^{(2)}\left(\sum_{j} w_{i j}^{(2)} h_{j}^{(1)}+b_{i}^{(2)}\right) \\
y_{i} & =\phi^{(3)}\left(\sum_{j} w_{i j}^{(3)} h_{j}^{(2)}+b_{i}^{(3)}\right),
\end{aligned}
$$

where $\phi^{(1)}$ and $\phi^{(2)}$ are the activation functions (which can be different for different layers).

\subsection{The Data}

\section{The independent variables}

The independent variables in the analysis include firm characteristics taken from the CRSP/Compustat database. The construction of firm characteristics and the notation we use to refer to them are taken from [Green et al., 2013] with some further cleaning. Detailed characteristics and their calculations are provided in the appendix. We work with CRSP stocks, identified by their PERMNO code. We restrict the analysis to shares with codes starting with ' 10 ' and ' 11 ', and corresponding to stocks that traded in either NYSE, AMEX or NASDAQ.

CRSP firm characteristics are merged with ESG data provided by OWL Analytics, which aggregates data from different providers to provide monthly updated ESG scores covering 12 primary categories. The scores of each category are aggregated into the main three scores $\mathrm{E}, \mathrm{S}$, and $\mathrm{G}$, before being averaged into a single ESG metric (ESG score). Stocks in the OWL Analytics database are identified by their ISIN, and their coverage starts in 04-2009.

ESG data and firm characteristics are merged to form our data set. As suggested in [Green et al., 2017], accounting data that are updated once a year are six months lagged with the present time. Characteristics that are calculated from returns such as volatility, one- and six-month momentum, beta, and bid-ask spread are lagged by one month, while ESG scores are lagged by two months.. The idea is that the characteristics are used only when they were available to investors. 
We exclude all stocks non-positive book-to-market ratios as well as the $0.05 \%$ smallest stocks. Finally, we keep only stocks with at least twelve consecutive dates. The resulting data set covers 462 months (from 1980-01-31 until 2018-06-30) with a total of 17,050 stocks and an average of 3738 stocks per date. Figure 2 shows the number of stocks available in the analysis for each time period along with the number of stocks with ESG data.

Figure 2: Number of stocks in time

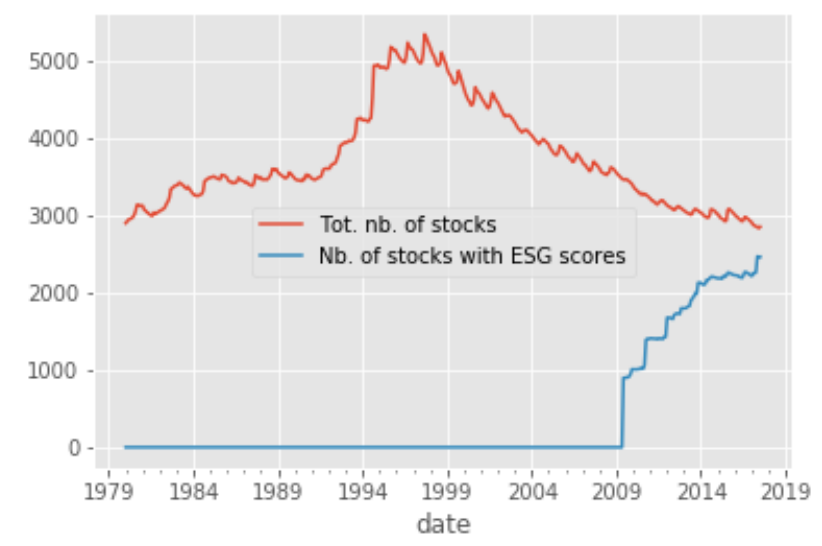

This figure reports the number of stocks available in the data set between January 1980 and June 2018 and the number of stocks with ESG scores.

\section{The dependent variable}

The maximum drawdown of a stock is defined as its largest cumulative loss from peak to trough over a fixed period $\tau$. Letting $P$ denote stock price, maximum drawdown can be calculated through the following formula:

$$
\operatorname{MDD}(P)=\sup _{t \in[0, \tau]} \sup _{s \in[t, \tau]}\left(\frac{P_{s}-P_{t}}{P_{t}}\right)
$$

Maximum drawdown is the largest drawdown a stock encounters on a given period and thus is unique for each period (and each stock). We rely on a one year forward maximum drawdown using the same moving window one would use to calculate annual returns. We display in Figure 3 two representation of $M D D$ : its evolution in time for two stocks and its cross-sectional distribution for two different periods.

Figure 3: One year forward Maximum Drawdown

The two figures report the evolution in time of the one year forward $M D D$ for IBM and BAC between 1980-01-01 and 2017-06-30 (left), and cross-sectional distribution for 2008-12-31 and 2014-12-31.
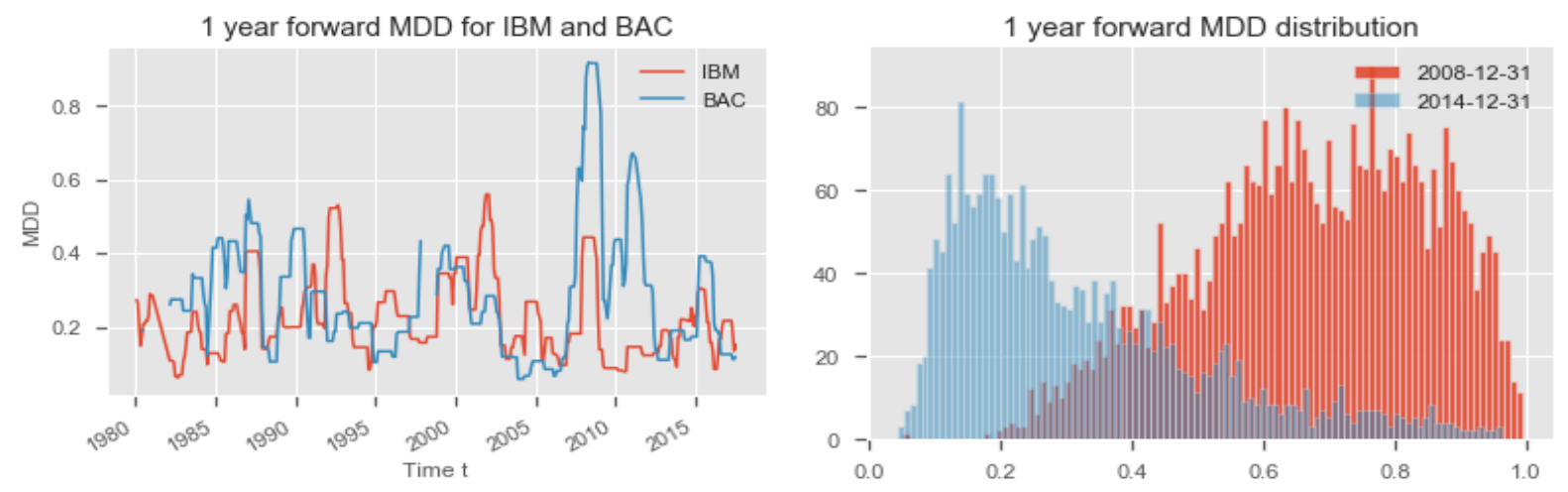


\section{Training, validation, and test data}

In financial economics, it is standard practice to split a dataset into in-sample and out-of-sample subsets and to evaluate performance based on the latter. For machine learning models, it is more common to split the data into three subsets: training, validation, and test. The training set is used to fit a number of models with the same general architecture but different hyperparameters. ${ }^{2}$ The validation set is used to tune the hyperparameters. The test set is used to evaluate model performance. This more elaborate procedure addresses the complexity of machine learning models, and their tendency to overfit or underfit when hyperparameters are poorly chosen 3

The avoidance of lookahead bias, the goal of subjecting our models to the most challenging tests possible, and the availability of data guide the specification of our training, validation, and test sets. When we run our models without ESG data, we use a training set that runs from 1980-01-01 until 1999-04-30, a validation set that runs from 1999-05-01 until 2007-03-31, and a training set that runs from 2007-04-01 to 2018-06-30. The short history of our ESG data, which begin on 2009-06-30, forces us to make two compromises. First, we skip the validation step and rely on the hyperparameters chosen in previous run (without ESG data). This leaves more data for testing. Second, we put the financial crisis in the training set instead of the test set, making it easier for our model to succeed. Our training set runs from 1980-01-01 until 2013-06-30 and our test set runs from 2014-07-01 to 2018-06-304

\section{Preprocessing}

Data preprocessing is the technique of transforming the raw data into an "understandable" format. Some machine learning algorithms are particularly sensitive to the range of values, and rescaling the data is a common way to overcome this issue. As we are interested in the cross-sectional impact of firm characteristics, we $z$-score the independent variables on each date. This means that for each date $t$, we subtract the cross-sectional average and divide by the cross-sectional standard deviation:

$$
X_{s c}=\frac{X-\mathbb{E}(X)}{\sigma(X)} .
$$

This transformation standardizes all variables to have mean 0 and variance 1 while maintaining the ranking of stocks by each variable along the cross-section.

Before running the models, we need to deal with missing data. This issue is pervasive and important in machine learning applications. Missing values in our data are caused by the absence of the information for a given stock at a given date. Removing stocks with missing information is not a solution as it will result in very few observations. Therefore, we rely on imputation methods. The most common imputation methods are mean, median, mode, and K-nearest neighbors. In our case, replacing missing values for categorical variables by their median and those in numerical variables by their mean gave better results on the validation set. Once again, the median and mean are taken over stocks on a given date.

\footnotetext{
${ }^{2}$ In machine learning, a hyperparameter is a parameter whose value is set before the learning process begins, and is specific to the model's architecture. Examples of hyperperameters are the penalization term for a penalized regression, and the number of hidden layers and number of units in a neural network. Hyperparameters are specified through a validation process and are chosen to minimize model error over the validation set.

${ }^{3} \mathrm{~K}$-fold cross-validation is a standard method for tuning hyperparameters. The method splits training data into $k$ equally sized subsets. A model with a fixed set of hyperparameters is fit on the union of $k-1$ subsets and evaluated on the $k$ th subset. This process is repeated $k-1$ times and the evaluation scores are averaged, yielding an overall score. This practice is, however, very costly and does not respect the time ordering of events, which is essential to our analysis.

${ }^{4}$ Since we evaluate maximum drawdown over one-year periods and stagger start dates by a month, a period of $N$ years contains $12 \times(N-1)$ observations.
} 


\subsection{Performance and feature importance}

Performance measures aim to evaluate a statistical model and are usually reported out-of-sample (OOS) data, i.e., on data that are not used to calibrate the model, to avoid overfitting. In the best case scenario, a model's prediction should be as close as possible to the real outcome. A measure of the error between the forecast and the actual value can be used to interpret the results. In our analysis, the non-stationarity of the dependent variable results in a different order of magnitudes between the prediction and actual value, leading to significant errors. Even though we might fall in such scenario, we can still have a relationship between the forecast and real value that we can observe on a scatter plot, and this relationship can be pointed by considering an "agreement" measure. The coefficient of determination, or R-squared, which measures the proportion of the variance in the dependent variable that is predictable from the independent variable, is commonly used for regression analyses. However, from our experience, R-squared is sensitive to the different magnitudes in the actual and predicted variable, in addition to outliers. We replace the latter by reporting two metrics; the mean absolute error, and Lin's concordance correlation coefficient. The first metric is a measure of accuracy and proportional to the $\mathcal{L}_{1}$ distance, while the second metric reports the degree of agreement between two variables, and is proportional to Pearson's correlation.

We recall the formula for the mean absolute error $(M A E)$ :

$$
M A E=\frac{1}{\# \mathcal{T}} \sum_{(i, t) \in \mathcal{T}}\left|y_{i, t+1}-\widehat{y}_{i, t+1}\right|
$$

where $\mathcal{T}$ denotes the test sample.

$M A E$ measure is more robust to outliers than squared error and the values of $M A E$ are on same scale as the data. It is a measure of the error so the model with smallest MAE is the the better in terms of approximating the magnitude of the drawdown.

Lin's concordance correlation coefficient (CCC), see [Lin et al., 2002], is a measure of the agreement between two variables defined by:

$$
C C C=1-\frac{\mathbb{E}\left[(y-\hat{y})^{2}\right]}{\mathbb{E}\left[(y-\hat{y})^{2} \mid \rho=0\right]}
$$

Lin's $C C C$ uses the mean squared error $(M S E)$, i.e., $\mathbb{E}\left[(y-\hat{y})^{2}\right]$, and a conditional version of it, i.e, $\mathbb{E}\left[(\boldsymbol{y}-\hat{y})^{2} \mid \rho=0\right]$ which measures the squared distance in the absence of relationship between the two variables $(\rho=0)$, to obtain an agreement coefficient between -1 and 1. A perfect match is achieved when $C C C=1$, while $C C C=0$ would mean no relationship between the prediction and realized value.

Numerically, the value of $C C C$ is estimated from a sample by:

$$
\widehat{C C C}=\frac{2 \rho \sigma_{y} \sigma_{\hat{y}}}{\left(\mu_{y}-\mu_{\hat{y}}\right)^{2}+\sigma_{y}^{2}+\sigma_{\hat{y}}^{2}},
$$

where $\sigma_{y}^{2}$ is the variance of $y$ and $\rho$ Pearson's correlation between $y$ and $\hat{y}$. As for $M A E$, the measure is calculate on the test set $\mathcal{T}$.

The idea of feature importance is to measure how much a performance measure score decreases when a feature is not available. A potential solution would be to remove the feature from the data set, re-train the model with the other estimators, and measure the performance. On the one hand, this solution requires re-training and can be computationally intensive. On the other hand, it shows what feature is important within the data set rather than within a given trained model. Finally, while 
some models allow having an implicit measure of the importance of a given variable, e.g., regression coefficients for linear regression models, more complicated models like neural networks lack this characteristic.

To address feature importance on a unified framework, [Ribeiro et al., 2016] propose the "local interpretable model-agnostic explanations". Their method relies on local surrogate models that are trained to approximate the predictions of the underlying black box model. A surrogate model can be any interpretable model such as linear regression or a decision tree. In their analysis of the predictability of monthly returns, [Gu et al., 2018] measure the importance of a variable by the reduction in $R^{2}$ obtained by setting all the values of the selected variable to zero and keeping the values of the other variables fixed. [Wei et al., 2015] review and compare a large set of methods for variance importance models (VIM) including difference-based VIMs, hypothesis test techniques, or variance-based VIMs. The intuition behind these methods is that the more a model's decision criteria depend on a feature, the more the predictions change as a function of perturbing this feature. For our analysis, we rely on the method known as "permutation importance" by measure how score decreases when replacing the feature by a random noise drawn from the same distribution as the original feature values, see [Breiman, 2001] and [Altmann et al., 2010].

\section{Empirical Study}

We collect monthly data from WRDS and OWL Analytics for stocks listed in the NYSE, AMEX, and NASDAQ. After calculating one-year forward maximum drawdown, we obtain a data set that starts in January 1980 and ends in June 2017, totaling 450 months. We account for 96 characteristics in total, including 16 ESG scores, 5 binary variables, and 1 categorical variable with 9 outcomes for sectors (based on Bloomberg's categories).

The regression is performed by considering maximum drawdown as the dependent variable on one hand, and the matrix with rescaled firm characteristics as the predictors on the other hand. Rescaling the (raw) predictors to a common level addresses, in part, the non-stationarity of raw predictors, leading to improved out-of-sample results.

After training the nine models, i.e., linear model (LM), Lasso, Ridge, Elastic Net (ENet), Random Forest (RF), Partial Least Square (PLS), Principal Component Regression (PCR), Extreme Gradient Boosting Trees (XGBoost), and Multi-layer Perceptron (MLP), we compare the results based on the out-of-sample performance measures.

\subsection{The cross-section of maximum drawdown ${ }^{5}$}

\subsubsection{A first analysis without ESG data}

Because the ESG data begins in 2009, we omit ESG scores for a first analysis in which we test the models using the other 80 variables. This enables us to carry out the analysis on a larger test set that includes the 2008 crisis. The training set starts in January 1980 and ends in March 2006, while the test set to start in March 2007 and ends in June 2017.

\section{Performance}

In figure 4, we report MAE and CCC measures for all the stocks, and for the the top and bottom quartiles by market capitalization. The best performance was achieved by MLP (MAE $=14.21 \%$

\footnotetext{
${ }^{5}$ We also conduct a similar analysis for the one-year excess returns to the risk-free rate. While these results are not detailed in this paper, they are summarized in footnotes throughout the results section.
} 
and $C C C=51.28 \%)$, followed by XGBoost $(14.27 \%$ and $C C C=48.28 \%)$. The values of $M A E$ are relatively close from one model to another, while we notice a bigger gap in CCC measure across different models6.

Figure 4: Overall $M A E$ and $C C C$

The tables and figures in panels (a), (b) and (c) report out-of-sample MAE and CCC for the test period from 03-2006 until 06-2017 for all stocks, and for top and bottom quartiles of stocks sorted by market capitalization. Results are reported for nine models: ordinary least square (OLS), Lasso, Ridge, elastic-net (ENet), partial least square (PLS), principal component regression (PCR), random forest (RF), XGBoost, and multi-layer perceptron (MLP).

(a) Table of out-of-sample $M A E$

\begin{tabular}{lrrrrrrrrr}
\hline & OLS & Lasso & Ridge & ENet & PLS & PCR & RF & XGBoost & MLP \\
\hline all & 14.46 & 14.46 & 14.50 & 14.46 & 14.52 & 14.52 & 14.57 & 14.27 & 13.99 \\
top quartile & 13.24 & 13.24 & 13.34 & 13.24 & 13.27 & 13.22 & 13.20 & 13.13 & 12.47 \\
bottom quartile & 15.14 & 15.14 & 15.23 & 15.14 & 15.46 & 15.32 & 15.41 & 15.03 & 14.75 \\
\hline
\end{tabular}

(b) Table of out-of-sample CCC

\begin{tabular}{lrrrrrrrrr}
\hline & OLS & Lasso & Ridge & ENet & PLS & PCR & RF & XGBoost & MLP \\
\hline all & 46.71 & 46.68 & 46.14 & 46.69 & 46.75 & 46.52 & 46.77 & 48.65 & 50.53 \\
top quartile & 29.30 & 29.27 & 29.14 & 29.29 & 29.60 & 30.52 & 34.09 & 33.67 & 34.33 \\
bottom quartile & 52.57 & 52.54 & 51.71 & 52.55 & 51.97 & 51.68 & 47.92 & 51.83 & 54.17 \\
\hline
\end{tabular}

(c) Barplot of out-of-sample MAE (left) and CCC (right)
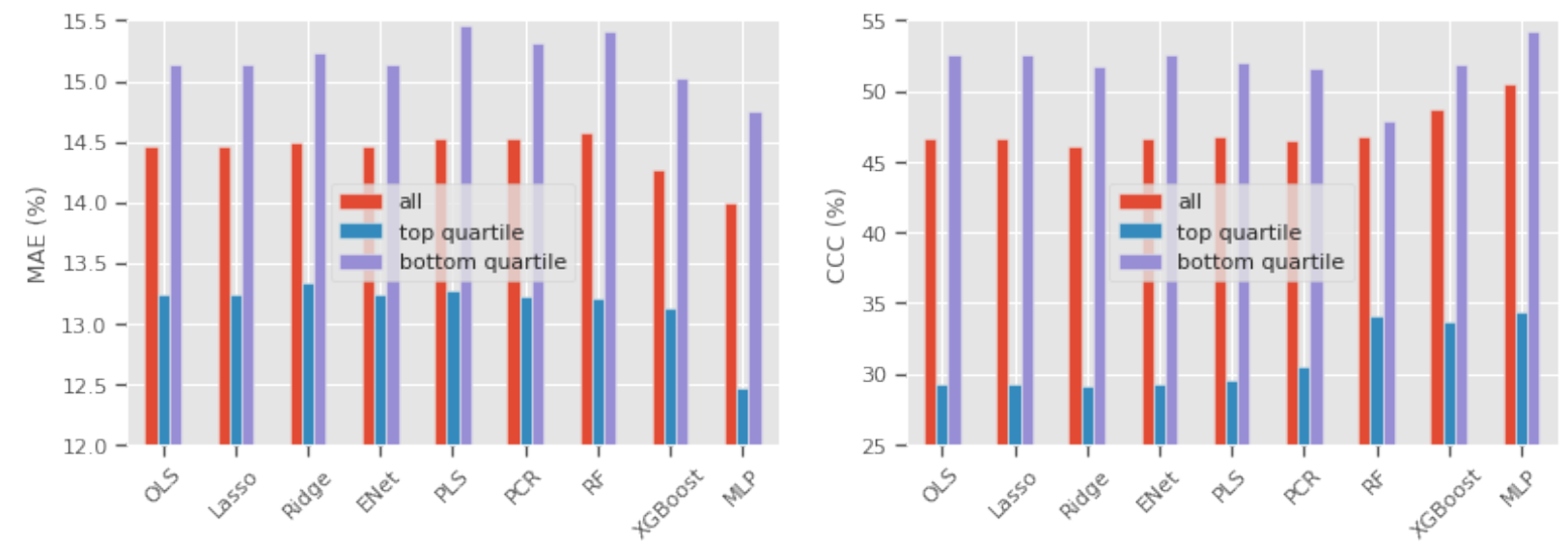

The previous results are aggregated over the entire sample period, and they do not inform us about when the models perform poorly and when they gave a sound estimation. To investigate this question, we calculate the performance measures over the cross-section for each date of the training period, leading to a time series of out-of-sample performance metrics. The results are shown in Figure 5. Without exception, all models performed poorly during the 2008 financial crisis. While the $M A E$ more than doubled relative to its usual values, CCC remained positive (slightly above $17 \%$ ). This value indicates that, despite the poor performance, the concordance between the predicted and realized maximum drawdown is persistent. To inspect this claim further, we divide our stocks for each date into 10 quantiles based on their predicted maximum drawdown. We then plot the empirical distributions of the stocks' realized drawdown 7

\footnotetext{
${ }^{6}$ For the case of one-year excess returns, $M A E$ is large because of the high noise to signal ratio. Looking at the concordance measure CCC, we find that non-linear models slightly outperform linear ones. Random forest has the best performance $(9.9 \%)$, followed by XGBoost $(8.86 \%)$, MLP $(7.78 \%)$ PLS $(8.01 \%)$, PCR $(7.5 \%)$ and OLS $(7.74 \%)$.

${ }^{7}$ Similar to maximum drawdown, the period around the 2008 crisis is where the performance is the poorest for all the models. CCC reaches negative values, while MAE attains $90 \%$. In mild market conditions, the value of CCC varies around 15\%, reaching 24\% around March 2015, while MAE varies around 35\%. Tree-based models exhibit a slightly better
} 
Figure 5: The evolution of MAE (left) and CCC (right) in time between 03-2007 and 06-2017
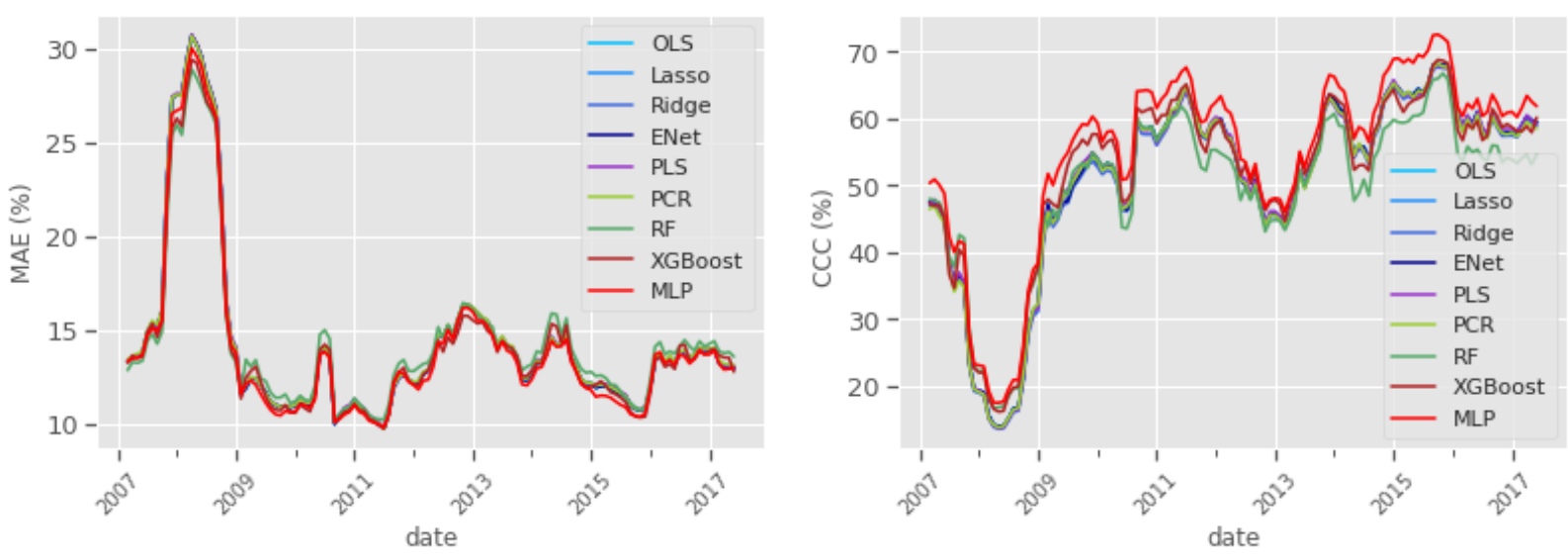

Figure 6: Realized $M D D$ distribution for predicted $M D D$-based quantiles

The quantiles $q 1, \ldots, q 10$ are formed using the empirical distribution of predicted $M D D$ for each of the two dates 2008-04-01 and 2015-04-01. The distribution of realized MDD is then displayed for each quantile over these periods for the MLP method.
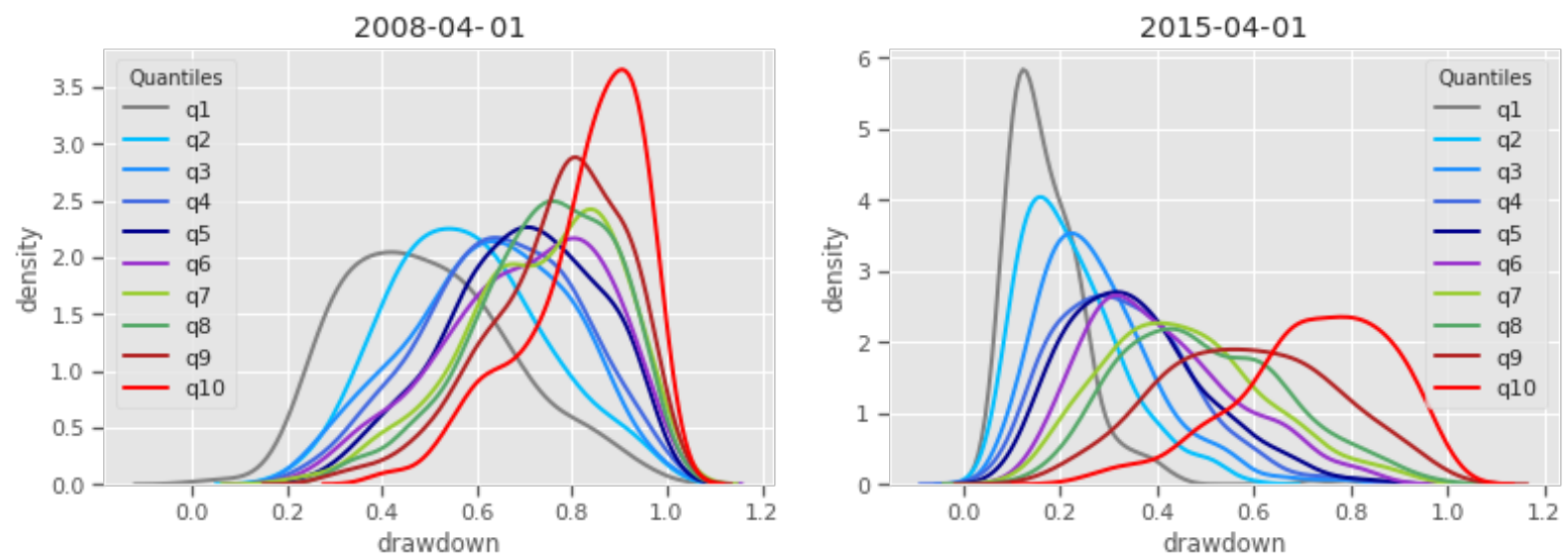

Figure 6 displays the relationship between predicted and realized maximum drawdown over two year-long periods. For each period, stocks are sorted into ten quantiles on the basis of predicted maximum drawdown, and the distributions of realized maximum drawdown are plotted. The first period (between 2008-04-01 and 2009-03-31) includes the financial crisis, while calmer market conditions prevailed during the second period (between 2015-04-01 and 2016-03-31). During the calmer period, the densities of different quantiles are more distinct. However, during the crisis, the middle quantiles tend to overlap, however, extreme quantiles remain well distinguished 8

\section{Feature importance}

To explore the predictive power of different firm characteristics, we calculate a feature importance score for the top 20 variables in each model over the test period. Reported in Figure 7, these results show that, on average, size (mve), volatility (retvol), bid-ask spread (baspread), beta, and volume (dolvol) were the dominant factors. While features identified as "important" tended to be consistent

performance than other models.

${ }^{8}$ The same analysis for excess returns to the risk-free rate shows less distinction between different quantiles but the lower quantiles have an average return that is lower than the higher quantiles, except in post 2008 crisis where the order is inverted. 
across models, there was (naturally) some variation. For example, dividend yield (dy) was exceptionally important for XGBoost, while size was exceptionally low compared to other models. The sign of the relationship between the predictors and the outcome, predicted $M D D$, is also informative. For example, our results suggest that larger companies, and companies with higher traded volume (higher dolvol) tended to have lower drawdowns, while companies with high volatility, bid-ask spread, or beta tended to have higher drawdowns.

Figure 7: Feature importance for different models

The figure reports feature importance defined by sensitivity of the explained variance. The values are reported for each of nine models over the out-of-sample period between 03-2007 and 06-2017. We differentiate between a negative relationship (red) and positive relationship (black) between the predictor and the output.
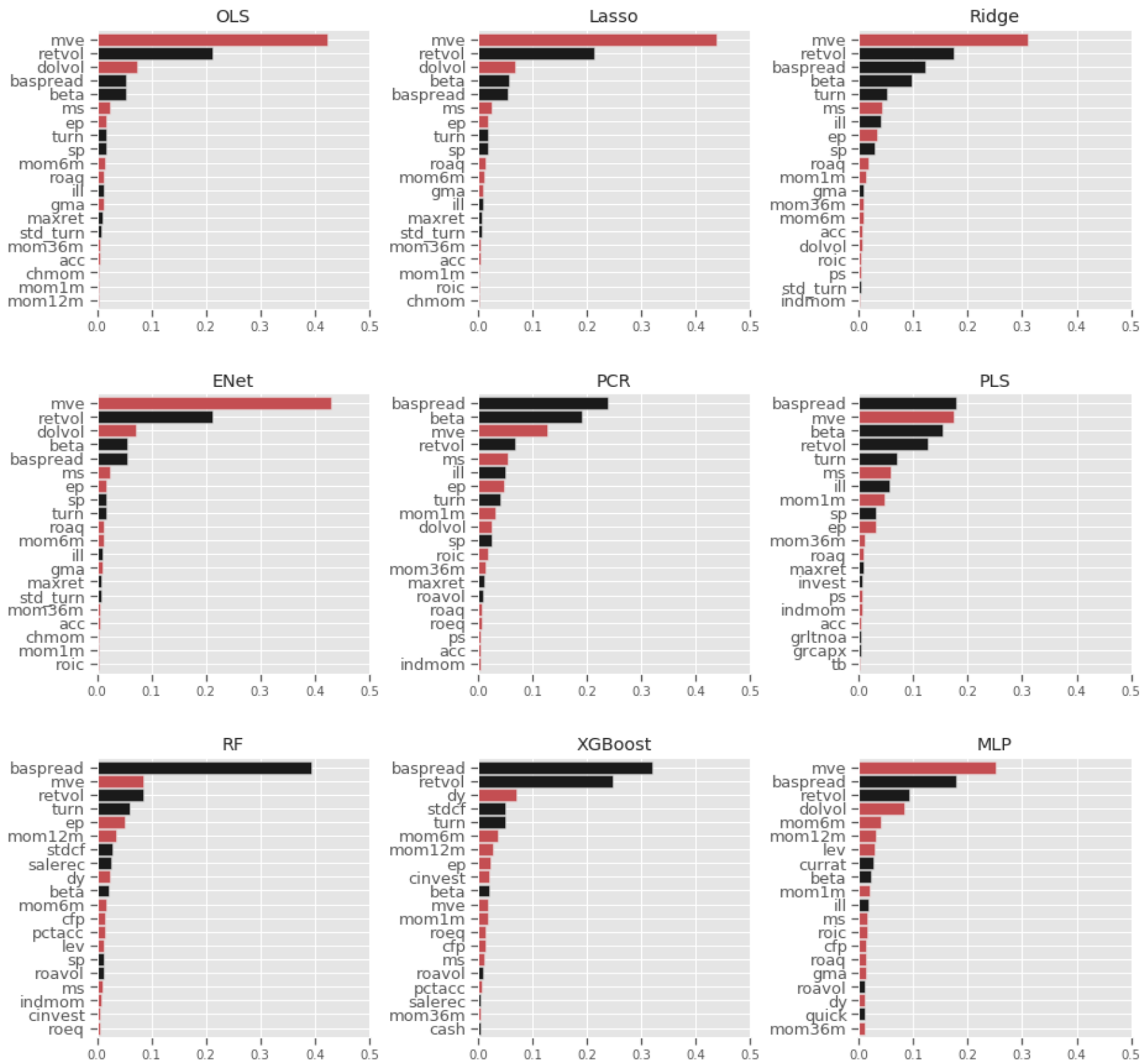


\subsubsection{The impact of ESG data on the cross-section of maximum drawdown}

Having investigated the ability of non-ESG firm characteristics to predict maximum drawdown on a large test set, we repeat the analysis, adding ESG to the mix. Our goal is to determine whether indicators of sustainability improve our ability to predict maximum drawdown. Since the ESG data set begins only in 2009, we are forced to extend our training set to include the finanicial crisis, making it easier for the models to perform well. The period 01-1980 until 06-2013 is thus used to train the models, and the period from 06-2014 until 06-2017 is used to test their performance.

Due to the limitations of linear models with regard to missing values, we restrict the analysis to non-linear models; i.e., random forest, XGBoost, and multi-layer perceptron. We compare the results based on the out-of-sample mean absolute error $(M A E)$ and Lin's concordance correlation coefficient (CCC) as before. For this case, we test for two alternatives of firm characteristics; the 80 features used in the previous analysis, and a trimmed version with common used variables (a total of 12). We also separate the 16 ESG variables into three cases. Notations and definitions of these cases are as follows:

- FC: All firm characteristics (80) without ESG scores.

- trimmed-FC: A trimmed set of twelve firm characteristics: sector, book-to-market (bm), volatility (retvol), beta, size (mve), return of equity (roeq), return on asset (roaq), momentums (mom $1 \mathrm{~m}$, mom $6 \mathrm{~m}$, mom $12 \mathrm{~m}$ and $\mathrm{mom} 36 \mathrm{~m}$ ) and earnings-to-price (ep).

- refined-E/S/G: Non-aggregated environmental, social, and governance scores totaling 12 scores.

- E/S/G: The main three environmental (E), social (S), and governance (G) scores which aggragate the previous twelve score.

- ESG: The single ESG score which averages the previous E, S, and G scores.

\section{Performance}

In figure 8, we report $M A E$ and $C C C$ for random forest (RF), XGBoost, and multi-layer perceptron (MLP). We distinguish between the 8 cases detailed earlier. We benchmark the new results against the results of the previous section (with the extended test set) in order to gauge the impact of the choice of test period. Performance was better on the shorter test set. This outcome is not surprising as the shorter test set excludes the financial crisis. The model with the lowest performance was RF: MAE ranged from $12.19 \%$ to $13.14 \%$ and CCC ranged from $56.94 \%$ to. $61.11 \%$ Both XGBoost and MLP provide better performance than RF. MAE values were lower for XGBoost $(11.41 \%$ vs. $11.90 \%$ in "trimmed-FC + E/S/G" case), however, CCC is higher for MLP $(64.19 \%$ vs. $62.04 \%$ in "FC + $\mathrm{E} / \mathrm{S} / \mathrm{G}$ ") when including all firm characteristics. These results suggest that XGBoost was more prone to overfitting on a larger of predictors. Overall, ESG variables improved the performance of both models. The best performance for XGBoost was "trimmed-FC + ESG" (11.36\% MAE and 63.35\% CCC ), and "FC + E/S/G" for MLP (11.79\% and $64.19 \%$ respectively)

\footnotetext{
${ }^{9}$ For excess returns, CCC for the base case (without ESG scores) varies around 14\%. It increases when accounting for ESG scores by around 3\% in absolute value (15\% in relative value). The different in performance between the aggregate ESG metric and the more detailed scores is very small. MAE on the other hand, varies around 33\% and decreases slightly for XGBoost when accounting for ESG data.
} 
Figure 8: Overall $M A E$ and $C C C$

The table and barplots report the overall $M A E$ and $C C C$, and cover non-linear models, i.e., random forest (RF), XGBoost, and multi-layer perceptron (MLP). We differentiates between two cases in choosing firm characteristics without ESG variables; FC for all the characteristics and trimmed-FC for twelve characteristics commonly selected in the industry, and four cases in selecting the set of ESG variables to add to the characteristics. This results in eight different cases reported in the analysis. Except from FC0, all cases use a test period from 06-2014 until 06-2017, while FC0 corresponds to the previous test period from 03-2007 until 06-2017.

(a) Table of out-of-sample MAE and CCC

\begin{tabular}{lccccccc}
\hline & \multicolumn{3}{c}{ MAE } & & \multicolumn{3}{c}{ CCC } \\
\cline { 2 - 4 } \cline { 6 - 8 } & RF & XGBoost & MLP & & RF & XGBoost & MLP \\
\hline FC0 & 14.57 & 14.57 & 14.57 & & 46.77 & 46.77 & 46.77 \\
FC & 14.27 & 14.27 & 14.27 & & 48.65 & 48.65 & 48.65 \\
FC + refined-E/S/G & 13.99 & 13.99 & 13.99 & & 50.53 & 50.53 & 50.53 \\
FC + E/S/G & 12.84 & 11.83 & 11.91 & & 58.35 & 62.04 & 62.78 \\
FC + ESG & 12.89 & 11.72 & 11.82 & & 57.95 & 62.20 & 63.71 \\
trimmed-FC & 13.33 & 12.93 & 13.12 & & 57.83 & 59.49 & 59.80 \\
trimmed-FC + refined-E/S/G & 12.19 & 11.44 & 12.42 & & 61.14 & 63.31 & 61.19 \\
trimmed-FC + E/S/G & 12.23 & 11.41 & 12.38 & & 61.17 & 63.35 & 61.56 \\
trimmed-FC + ESG & 12.27 & 11.36 & 12.06 & & 61.11 & 63.29 & 61.54 \\
\hline
\end{tabular}

(b) Barplot of out-of-sample MAE (left) and CCC (right)
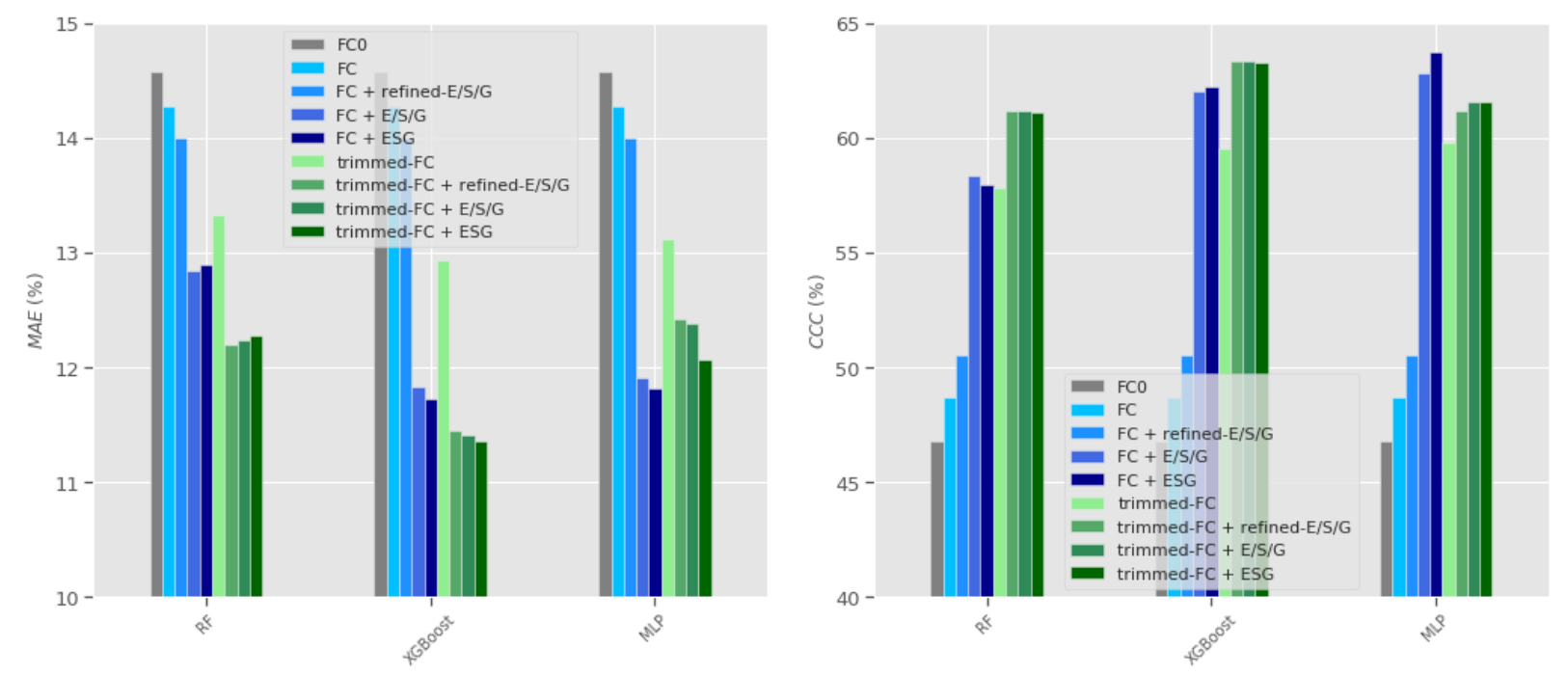

To explore how the models performed on different dates, we calculate the MAE and CCC measures across stocks for each date of the test set between 06-2014 and 06-2017. The time series of performance metrics for the multi-layer perceptron are shown in Figure 9. We find that excluding ESG variables (light blue and green bars) led to outperformance only during portions of 2015. Overall, including ESG variables improves the performance. The increase in performance using ESG variables is very similar when using the refined ESGs and when using the single average ESG score ${ }^{1011}$

\footnotetext{
${ }^{10}$ The results are similar for the two remaining models, random forest and XGBoost, and can be communicated upon request

${ }^{11}$ For excess returns, we find that the period of 2015 through 2016 has the best performance and decreases in 2017. Using all firm characteristics slightly outperforms the trimmed version, while ESG data improved the "trimmed-FC" case and to a lesser extent the "FC" case.
} 

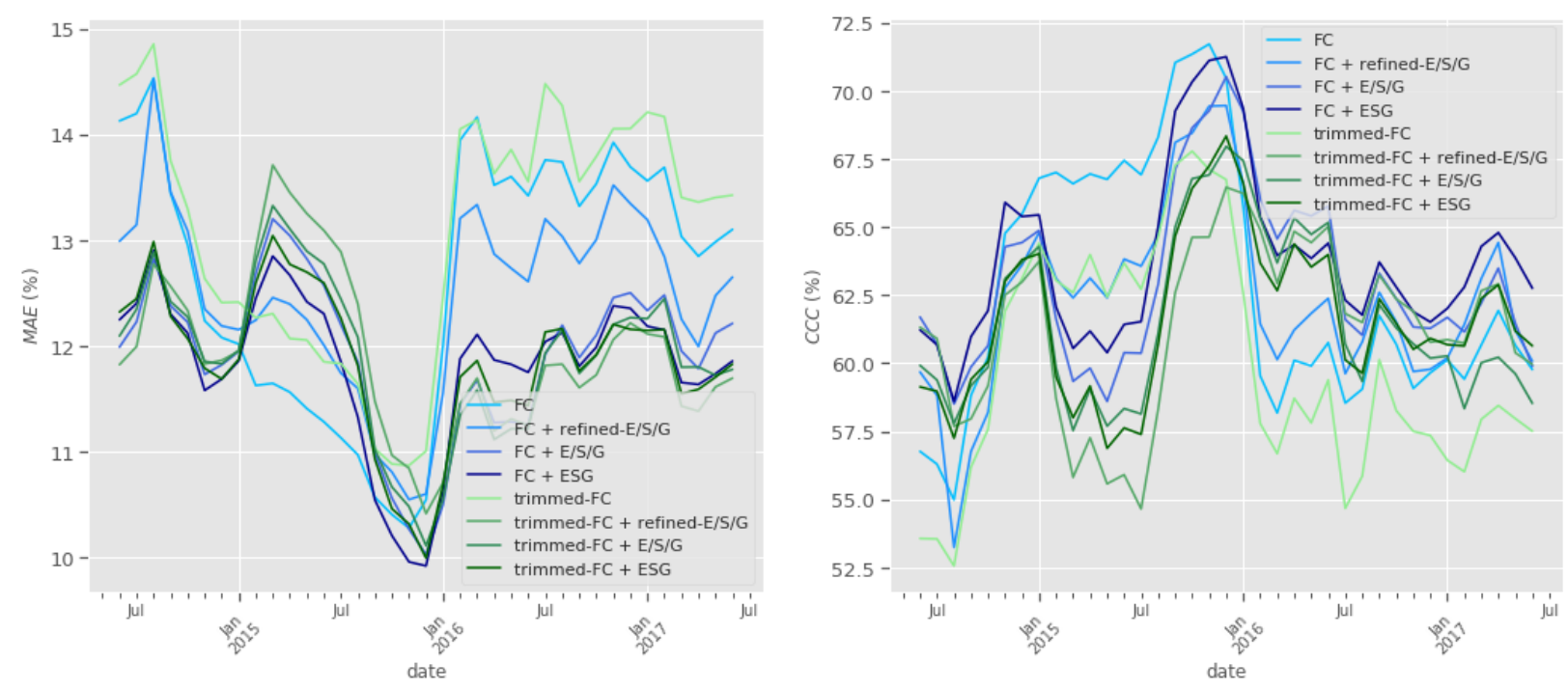

\section{Feature importance}

We repeat the feature importance analysis for the cases "FC + ESG" and "trimmed-FC + ESG" in order to assess the predictive power of ESG variables in non-linear models (RF, XGBoost and MLP). Figure 10 reports the top 30 variables and their importance scores given by their sensitivity for "FC + ESG". The leading dominant variables across the three models were bid-ask spread (baspread), volatility (retvol), earnings-to-price (ep), momentum (mom $12 \mathrm{~m})$ and beta. ESG score is also among the top 20 variables and ranks 3rd for XGBoost and 7th for MLP. This result is inline with the improvement in performance when including the score. When reducing the number of characteristics, i.e. "trimmed-FC + ESG", on average, we find volatility (retvol), size (mve), beta, earnings-to-price (ep), and momentum (mom $12 \mathrm{~m}$ ) were the top 5 variables, followed by the ESG single score. As noted above, the sign of the relationship is also informative. For example, companies with higher bid-ask spread, volatility or beta tended to have higher drawdowns, while larger companies with higher momentum or earnings-to-price tended to have lower drawdowns. We also find that high ESG scores were associated with lower drawdowns. This result is consistent across the tree-based models and the MLP model 12

\footnotetext{
${ }^{12}$ Some other configurations of MLP have led to a positive relationship between the ESG variable and maximum drawdown. However, these configurations were not optimal, based on the parameter tuning procedure. This discrepancy may be the result of colinearity of variables or the overfitting.
} 
Figure 10: Variable importance for case "FC + ESG"

The barplots report the top 30 variables for case "FC + ESG" and the three non-linear models; random forest (RF), XGBoost, and multi-layer perceptron (MLP). We differentiate between a negative relationship (red) and positive relationship (black) between the predictor and the output.
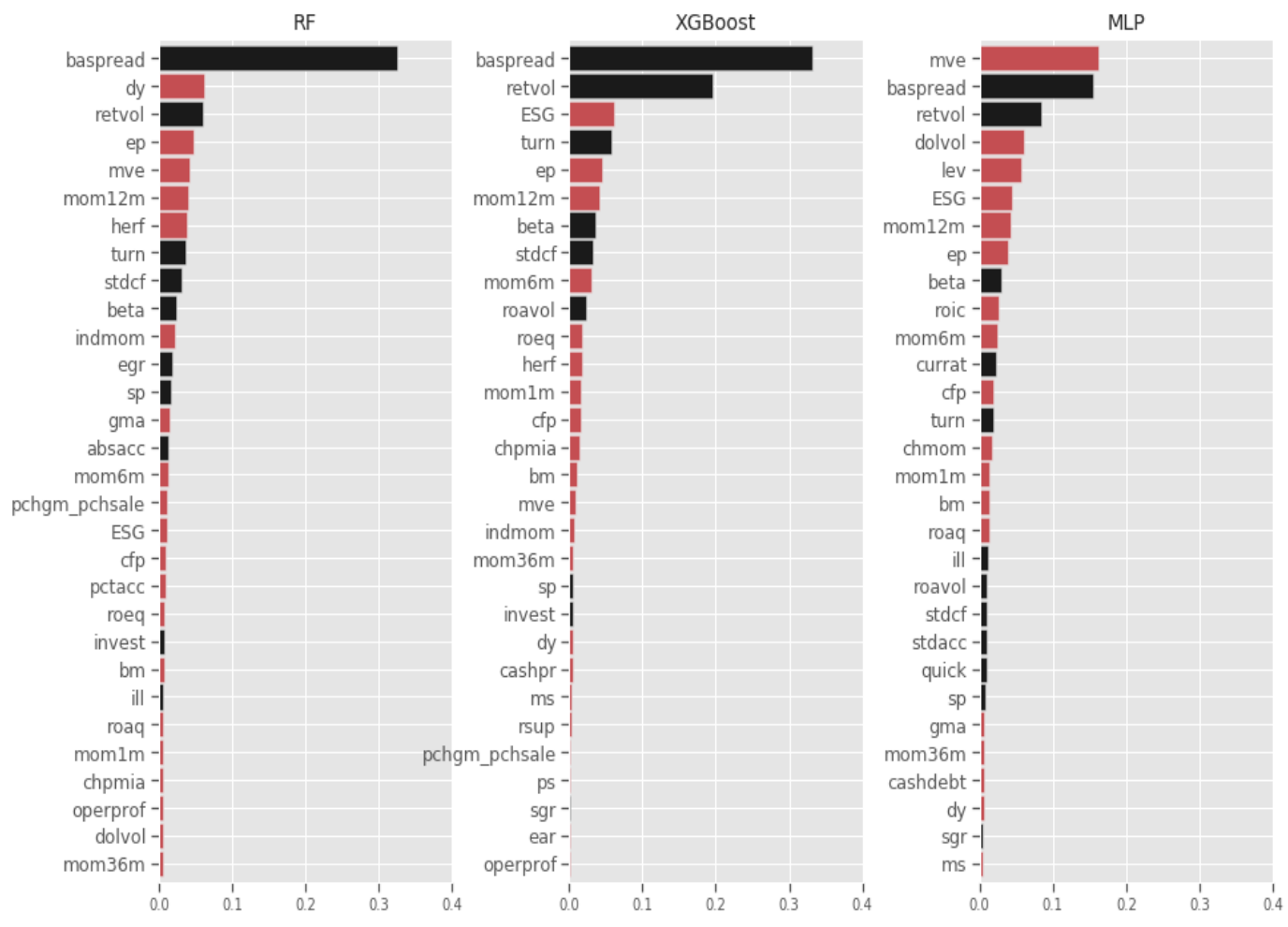

Figure 11: Variable importance for case "trimmed-FC + ESG"

The barplot reports variable importance for case "trimmed-FC + ESG" and the three non-linear models; random forest (RF), XGBoost, and multi-layer perceptron (MLP). The left panel presents the rank for each model and the sign of the relationship between the predictor and outcome:black for positive and red for negative. The right panel presents the rank by the average importance for different models and the sign of the relationship between the predictor and outcome: hatched for negative.
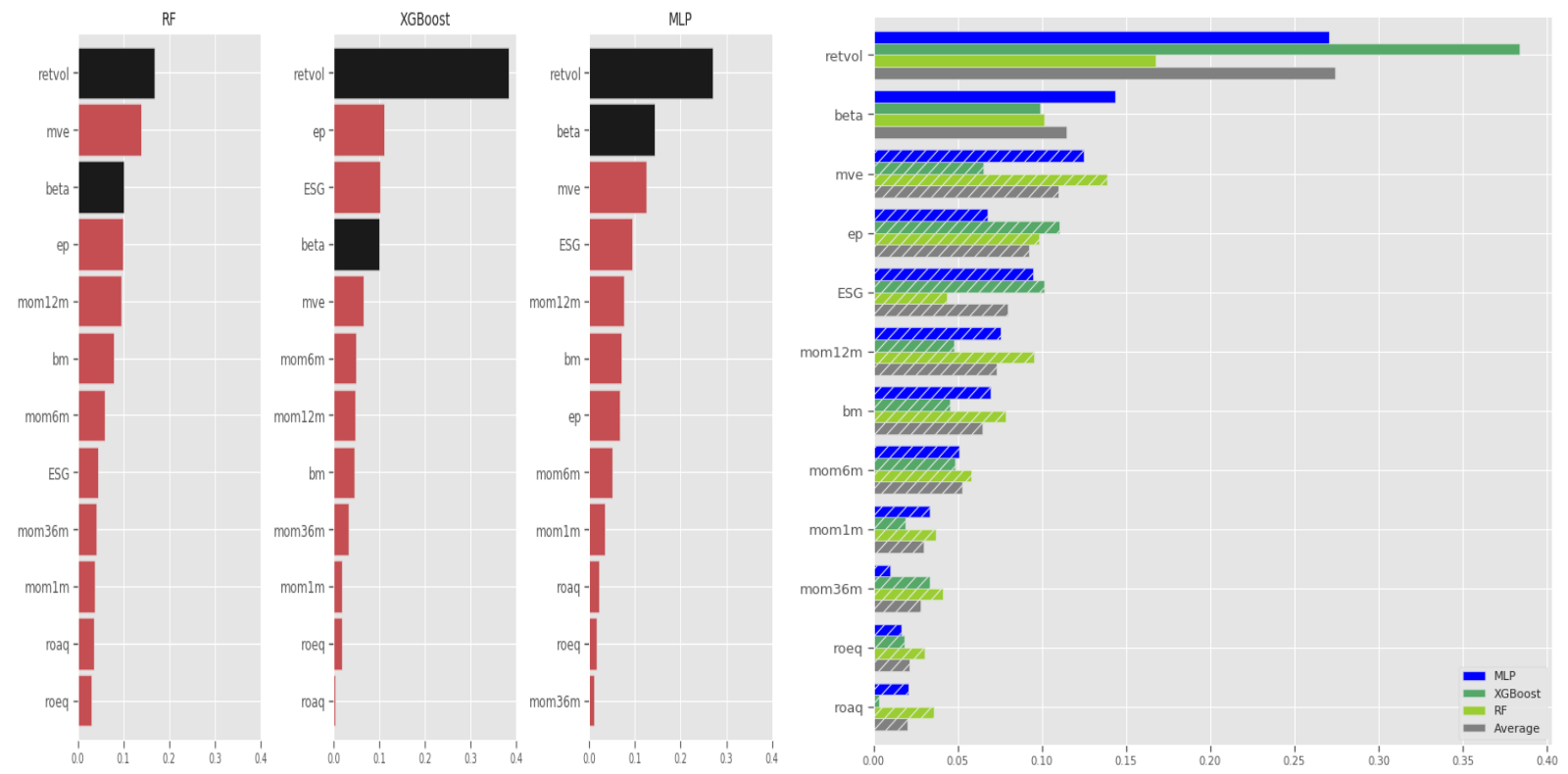


\section{Conclusion}

In this paper, we explore the cross-section of maximum drawdown in the US equity market. We apply a set supervised learning methods to the problem of forecasting the one-year maximum drawdown using current information on firm-level characteristics. The pooled regression approach we use indicates that non-linear methods outperform linear methods. We find that some firm characteristics can predict the cross-section of $M D D$. A number of these characteristics are consistently dominant across models, namely size, bid-ask spread, volatility, book-to-market, beta, and momentum. The agreement between predicted and realized maximum drawdown persists even in periods of turmoil. When including environmental, social, and governance scores to the set of predictors, we find improvement in the performance of non-linear methods. Finally, we find that the inclusion of a single aggregate ESG metric improved performance as much as the inclusion of more detailed scores, and that companies with a higher ESG metric were more likely to have lower drawdowns. 


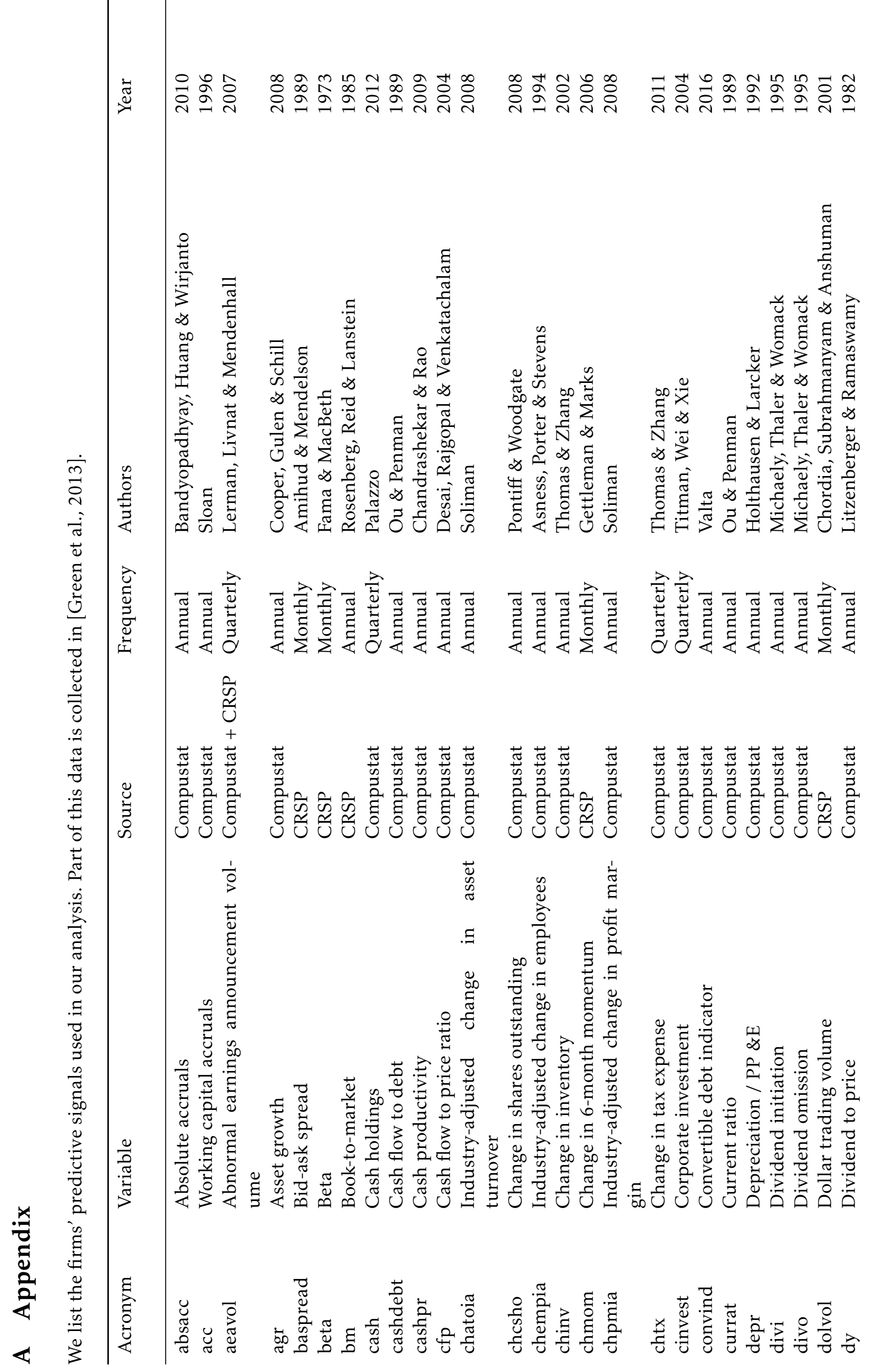




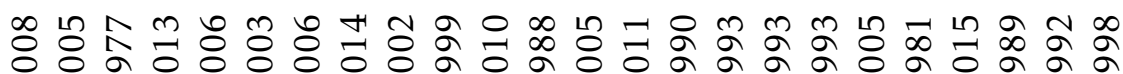

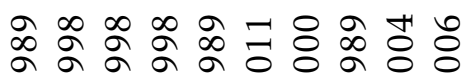

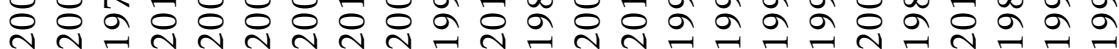

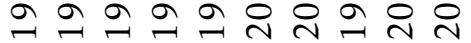

売

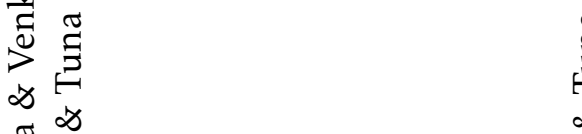

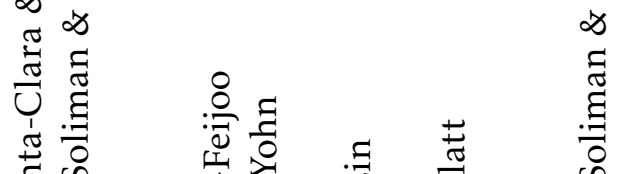

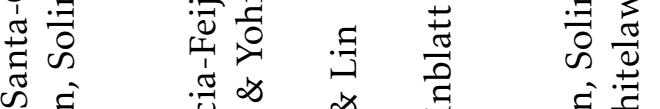

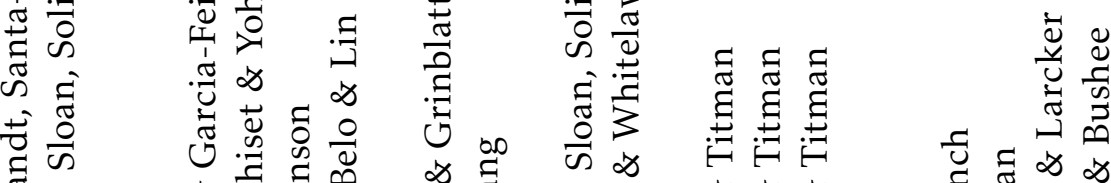
歾

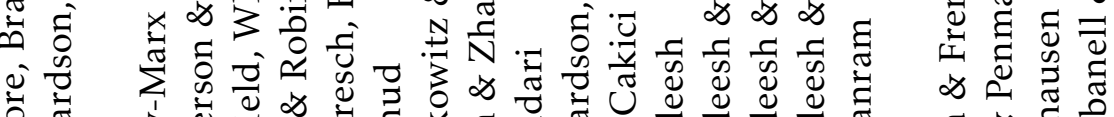

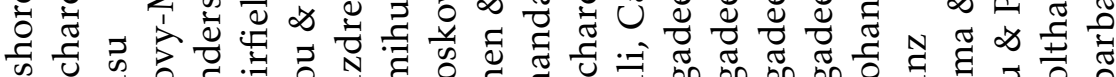

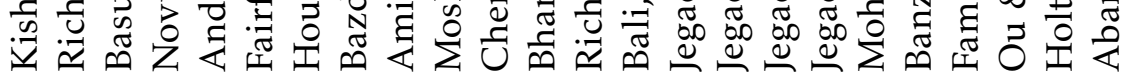

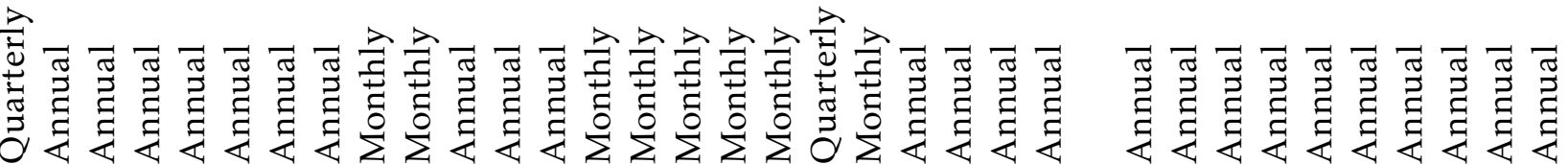

जิ

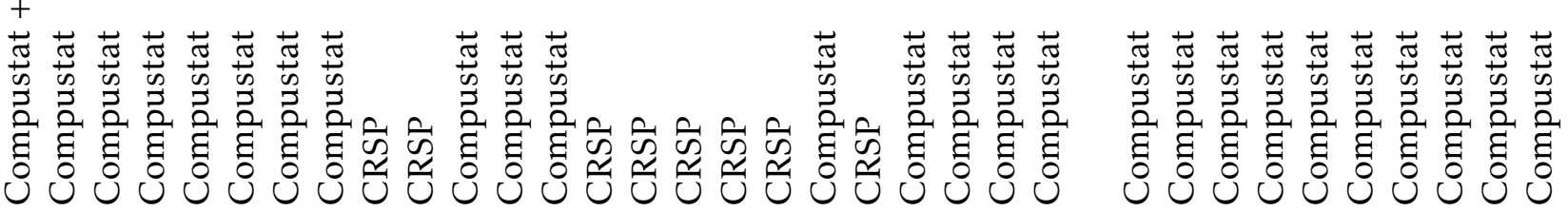

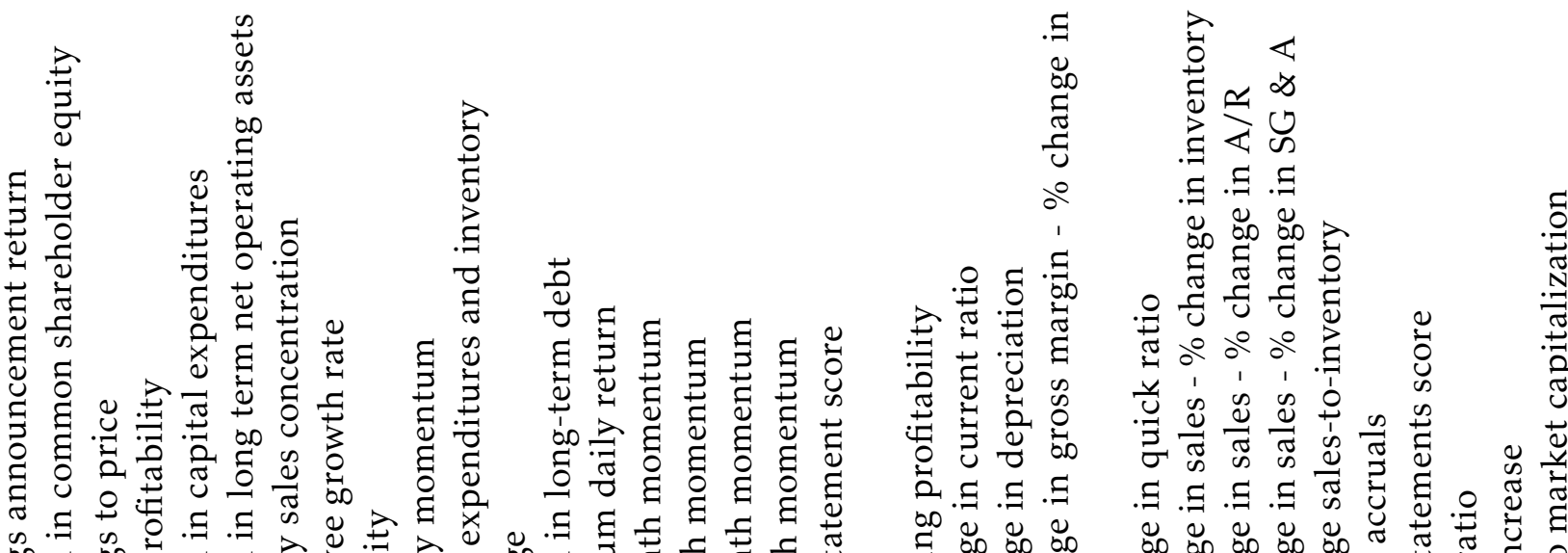

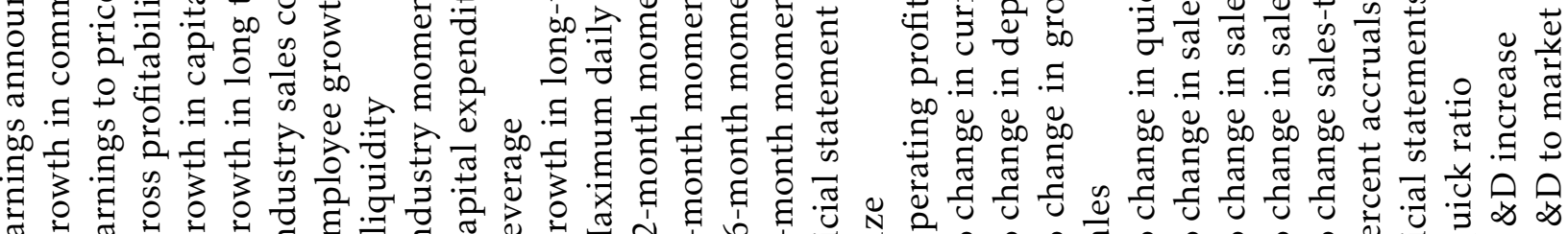

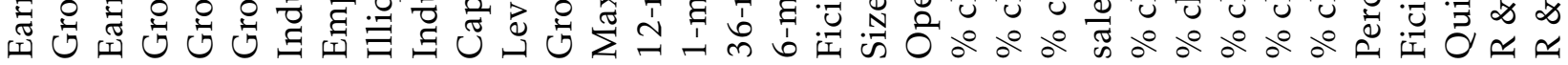

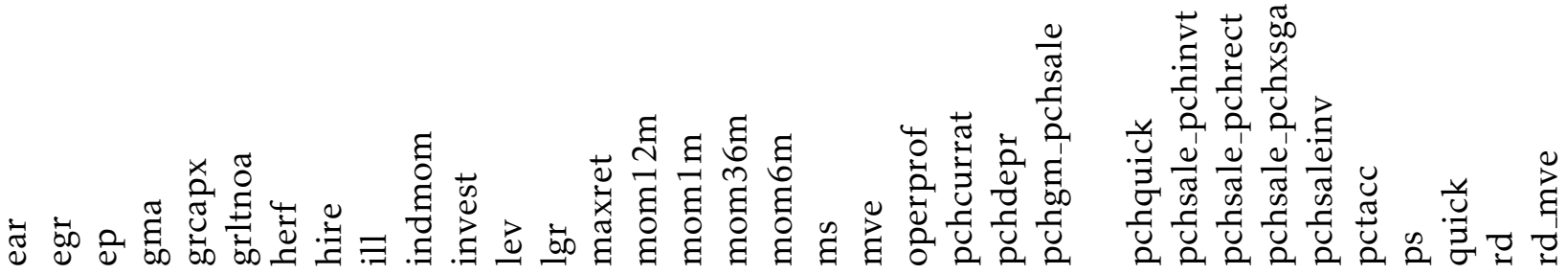




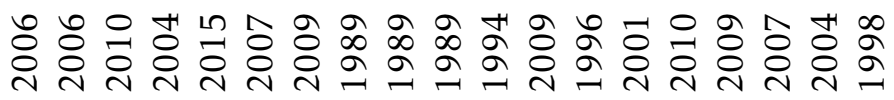

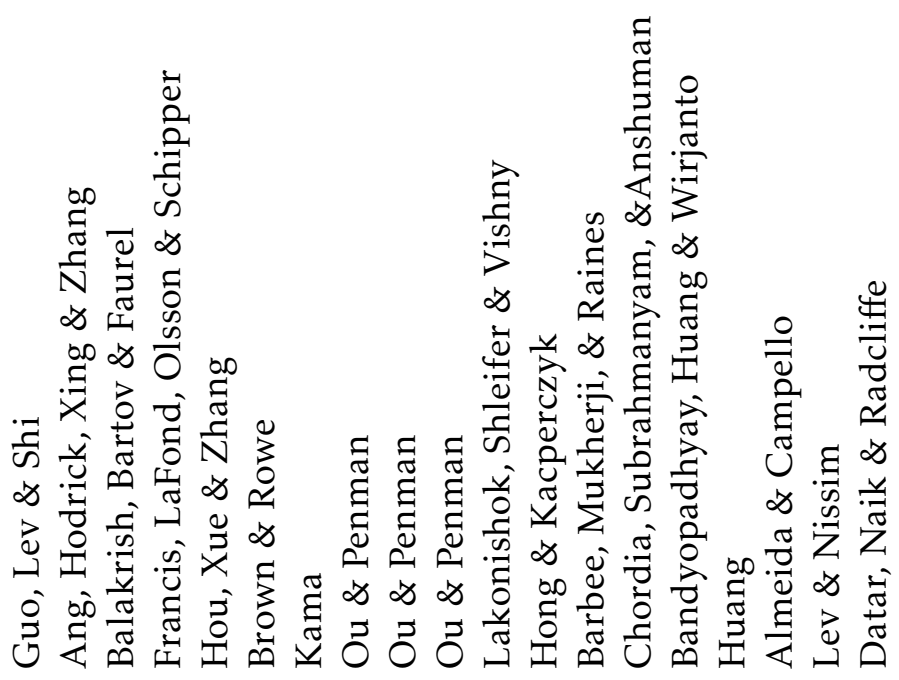

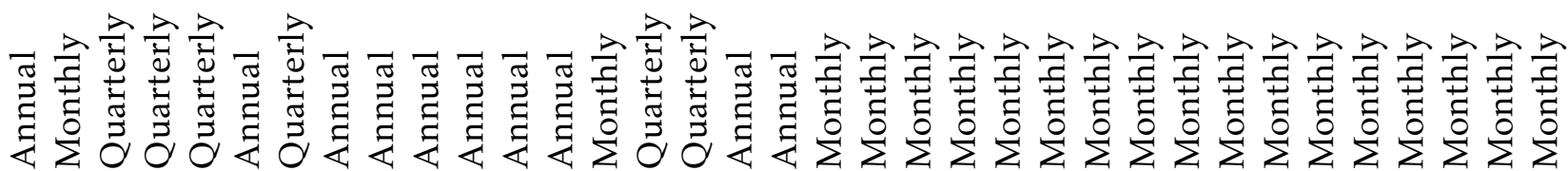

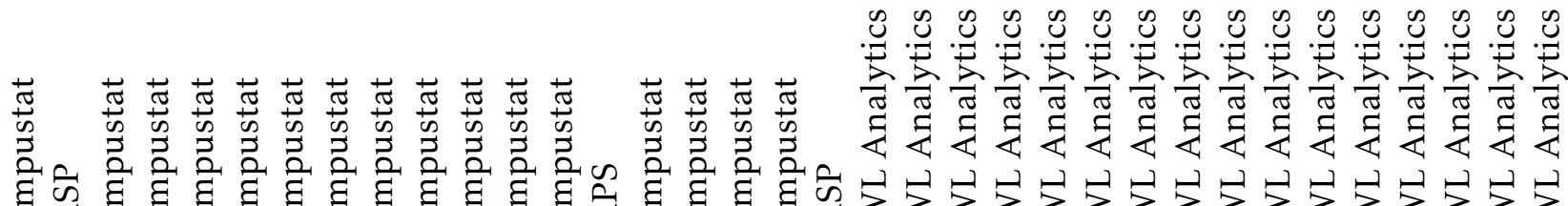

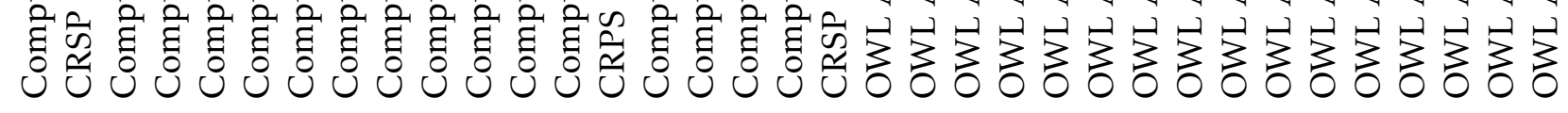




\section{References}

[Altmann et al., 2010] Altmann, A., Tolosi, L., Sander, O., and Lengauer, T. (2010). Permutation importance: a corrected importance measure. Bioinformatics, 26(10):1340-1347.

[Aupperle et al., 1985] Aupperle, K. E., Carroll, A. B., and Hatfield, J. D. (1985). An empirical examination of the relationship between corporate social responsibility and profitability. The Academy of Management Journal, 28(2):446-463.

[Bayraktar et al., 2014] Bayraktar, M. K., Mashtaler, I., Meng, N., and Radchenko, S. (2014). Barra US total market equity model for long-term investors. Technical report, MSCI.

[Benlemlih et al., 2018] Benlemlih, M., Shaukat, A., Qiu, Y., and Trojanowski, G. (2018). Environmental and social disclosures and firm risk. Journal of Business Ethics, 152(3):613-626.

[Brammer et al., 2006] Brammer, S., Brooks, C., and Pavelin, S. (2006). Corporate social performance and stock returns: Uk evidence from disaggregated measures. Financial Management, 35(3):97116.

[Breiman, 2001] Breiman, L. (2001). Random forests. Machine Learning, 45(1):5-32.

[Carhart, 1997] Carhart, M. M. (1997). On persistence in mutual fund performance. The Journal of Finance, 52(1):57-82.

[Chekhlov et al., 2004] Chekhlov, A., Uryasev, S., and Zabarankin, M. (2004). Portfolio optimization with drawdown constraints. In Supply Chain and Finance, pages 209-228. World Scientific.

[Chen and Guestrin, 2016] Chen, T. and Guestrin, C. (2016). XGBoost: A scalable tree boosting system. arXiv:1603.02754v1.

[Cholleta and Sandwidi, 2018] Cholleta, P. and Sandwidi, B. W. (2018). CSR engagement and financial risk: A virtuous circle? international evidence. Global Finance Journal.

[Daniel and Moskowitz, 2016] Daniel, K. and Moskowitz, T. J. (2016). Momentum crashes. Journal of Financial Economics, 122(2):221-247.

[Fama and French, 1992] Fama, E. F. and French, K. R. (1992). The cross-section of expected stock returns. The Journal of Finance, 47(2):427-465.

[Fama and French, 2008] Fama, E. F. and French, K. R. (2008). Dissecting anomalies. The Journal of Finance, 63:1653-1678.

[Feng et al., 2017] Feng, G., Giglio, S., and Xiu, D. (2017). Taming the factor zoo. Technical report, Fama-Miller Working Paper. Chicago Booth Research Paper No. 17-04.

[Goldberg and Mahmoud, 2016] Goldberg, L. R. and Mahmoud, O. (2016). Drawdown: from practice to theory and back again. Mathematics and FInancial Economics, 11:275-297.

[Green et al., 2013] Green, J., Hand, J. R. M., and Zhang, X. F. (2013). The supraview of return predictive signals. Review of Accounting Studies, 18(3):692-730.

[Green et al., 2017] Green, J., Hand, J. R. M., and Zhang, X. F. (2017). The characteristics that provide independent information about average U.S. monthly stock returns. The Review of Financial Studies, 12(1):4389-4436,.

[Gu et al., 2018] Gu, S., Kelly, B., and Xiu, D. (2018). Empirical asset pricing via machine learning. Working paper.

[Harvey et al., 2015] Harvey, C. R., Liu, Y., and Zhu, H. (2015). ... and the cross-section of expected returns. Review of Financial Studies, 29(1):5-68. 
[Heidorn et al., 2009] Heidorn, T., Kaiser, D. G., and Roder, C. (2009). The risk of funds of hedge funds: An empirical analysis of the maximum drawdown. The Journal of Wealth Management, 12(2):89-100.

[Herskovic et al., 2016] Herskovic, B., Kelly, B., and Lustig, H. (2016). The common factor in idiosyncratic volatility: Quantitative asset pricing implications. Journal of Financial Economics, 119(2):249-283. Journal of Financial Economics.

[Hoerl, 1962] Hoerl, A. E. (1962). Application of ridge analysis to regression problems. Chemical Engineering Progress, 58(3):54-59.

[Hong and Kacperczyk, 2009] Hong, H. and Kacperczyk, M. (2009). The price of sin: The effects of social norms on markets. Journal of Financial Economics, 93(1):15-36.

[Jo and Na, 2012] Jo, H. and Na, H. (2012). Does CSR reduce firm risk? Evidence from controversial industry sectors. Journal of Business Ethics, 110(4):441-456.

[Khan et al., 2016] Khan, M., Serafeim, G., and Yoon, A. (2016). Corporate sustainability: First evidence on materiality. The Accounting Review, 91(6):1697-1724.

[Lin et al., 2002] Lin, L., Hedayat, A. S., Sinha, B., and Yang, M. (2002). Statistical methods in assessing agreement: Models, issues, and tools. Journal of American Statistical Association, 97(457):257270.

[Lintner, 1965] Lintner, J. (1965). The valuation of risk assets and the selection of risky investments in stock portfolios and capital budgets. Review of Economics and Statistics, 47(1):13-37.

[Magdon-Ismail et al., 2004] Magdon-Ismail, M., Atiya, A. F., Pratap, A., and Abu-Mostafa, Y. S. (2004). On the maximum drawdown of a brownian motion. Journal of Applied Probability, 41(1):147-161.

[Pearson, 1901] Pearson, K. (1901). On lines and planes of closest fit to systems of points in space. Philosophical Magazine, 2(11):559-572.

[Plastira, 2014] Plastira, S. (2014). Performance evaluation of size, book-to-market and momentum portfolios. Procedia Economics and Finance, 14:481 - 490.

[Preston and O'Bannon, 1997] Preston, L. and O'Bannon, D. (1997). The corporate social-financial performance relationship: a typology and analysis. Business and Society, 36(4):419-429.

[Renneboog et al., 2008] Renneboog, L., Horst, J. T., and Zhang, C. (2008). Socially responsible investments: Institutional aspects, performance, and investor behavior. Journal of Banking $\mathcal{E}$ Finance, 32:1723-1742.

[Ribeiro et al., 2016] Ribeiro, M. T., Singh, S., and Guestrin, C. (2016). "why should I trust you?": Explaining the predictions of any classifierxplaining the predictions of any classifierexplaining the predictions of any classifier. KDD, ACM, pages 1135-1144.

[Sharpe, 1964] Sharpe, W. F. (1964). Capital asset prices: A theory of market equilibrium under conditions of risk. Journal of Finance, 19(3):425-442.

[Taylor, 1975] Taylor, H. M. (1975). A stopped Brownian motion formula. The Annals of Probability, $3(2): 234-246$.

[Tibshirani, 1996] Tibshirani, R. (1996). Regression shrinkage and selection via the Lasso. Journal of the Royal Statistical Society, 58(1):267-288.

[Vozlyublennaia, 2013] Vozlyublennaia, N. (2013). Do firm characteristics matter for the dynamics of idiosyncratic risk? Journal of International Financial Markets, Institutions E Money, 27:35-46. 
[Wei et al., 2015] Wei, P., Lu, Z., and Song, J. (2015). Variable importance analysis: A comprehensive review. Reliability Engineering and System Safety, 142:399-432.

[Zou and Hastie, 2005] Zou, H. and Hastie, T. (2005). Regularization and variable selection via the elastic net. Journal of the Royal Statistical Society, 67(2):301-320. 